\title{
Wavelength Conversion and 2R-Regeneration in Simple Schemes with Semiconductor Optical Amplifiers
}

\author{
Napoleão S. Ribeiro ${ }^{1}$, Cristiano M. Gallep ${ }^{2}$, and Evandro Conforti ${ }^{1}$ \\ ${ }^{1}$ Department of Microwave and Optics (DMO) -University of Campinas - Unicamp \\ ${ }^{2}$ Division of Telecommunication Technology (DTT) of FT/Unicamp \\ Brazil
}

\section{Introduction}

Future optical networks may require both wavelength conversion and bit shape regeneration in an all-optical domain. The possibility of pulse reshaping while providing wavelength conversion may support new demands over medium and large distances links (Kelly, 2001). Indeed, during propagation the optical data signal suffers deterioration due to the amplified spontaneous emission (ASE) from optical amplifiers, pulse distortion from intrinsic dispersion, crosstalk, and attenuation. All-optical regenerators may be important components for the restoration of these signals, providing complexity and cost reductions with the avoidance of optoelectronic conversions. The regeneration could be $2 \mathrm{R}$ (reamplification and reshaping) or 3R, which also provide retiming to solve jitter (Simon et al., 2008). Several $3 R$ regenerators using the semiconductor optical amplifier (SOA) have been proposed, such as cascaded SOAs setups (Funabashi et al., 2006) or SOA based MachZehnder interferometers (MZI) (Fischer et al., 1999).

However for small and medium distances systems, where the signal amplitude noise and distortions form the main problem and where jitter has fewer magnitudes, the simpler $2 \mathrm{R}$ processes can be adequate to keep signal quality (Simon et al., 1998). In addition, the SOA is a helpful device for both 2R-regeneration (Ohman et al., 2003) and wavelength conversion (Durhuus et al., 1996). Several techniques for 2R-regeneration based on SOAs have been proposed and tested, for example by using four-wave mixing (FWM) (Simos et al., 2004), cross-gain modulation (XGM) (Contestabile et al., 2005), integration within MZI (Wang et al., 2007), multimode interferometric SOA (Merlier et al., 2001), cross-phase modulation (XPM) with filtering (Chayet et al., 2004), and feed forward technique (Conforti et al., 1999). However, these techniques require complex designs and involve critical operation points, even the simplest ones based on XGM features. In addition, most of these techniques are not capable of wavelength conversion and regeneration simultaneously.

Recently a regenerator based on cross-gain modulation was proposed using one SOA for wavelength conversion (in a counter-propagating mode) and another deeply saturated SOA (synchronized by an optical delay line) to achieve cross-gain compression (Contestabile 2005). This efficient approach has similarities with the all-optical feed-forward techniques. In addition, this regenerator could not done wavelength conversion if the wavelength of the

Source: Advances in Lasers and Electro Optics, Book edited by: Nelson Costa and Adolfo Cartaxo,

ISBN 978-953-307-088-9, pp. 838, April 2010, INTECH, Croatia, downloaded from SCIYO.COM 
input signal is chosen in the output. Although good results can be obtained, this technique demonstrates to be complex since it used optical delay line and two SOAs. In this chapter, we introduce a more simple technique based on XGM (using just one SOA, an optical isolator, an optical circulator, and a CW laser) with easy robust operation at high speed reconfiguration (Ribeiro et al., 2008; Ribeiro et al., 2009a). The regeneration is based on the abrupt profile of the SOA cross-gain modulation efficiency, which is compressed at high input optical powers. The two optical carriers are amplified in the counter-propagating mode allowing conversion to another or to the same wavelength.

In addition, we present 2R-regeneration and conversion results for different kinds of deteriorated input signals. Experimental results such as eye diagrams and measured Qfactors are also shown, for various optical input powers, carriers detuning, bit rates and optical polarizations. Moreover, the estimative of the bit error rates (BER) are presented. Finally, the regenerator extinction ratio (ER) deterioration and its relation with the Q-factor improvements are discussed.

\section{Experimental setup}

The single-SOA all-optical 2R-regenerator setup is presented in Figure 1. This regenerator will be called $2 \mathrm{R}$-converter. The experimental scheme is divided in blocks. In the first block, the optical carrier at $\lambda_{1}$ is modulated by pseudo random bit sequence (PRBS) data. In most cases a non-return to zero (NRZ) modulation was used, and the polarization of the input signal was controlled to maximize de modulator response.

In the Deterioration Block, the signal was degenerated by different types of deterioration processes to analyze the regenerative effects of this device. In Figure 1, between point 1 and 2 , the three elements used to deteriorate the input signal are presented: another SOA as a booster, a buried fiber link (KyaTera-Fapesp Project) and an erbium doped fiber amplifier (EDFA). Different deterioration cases were obtained by combining these elements.
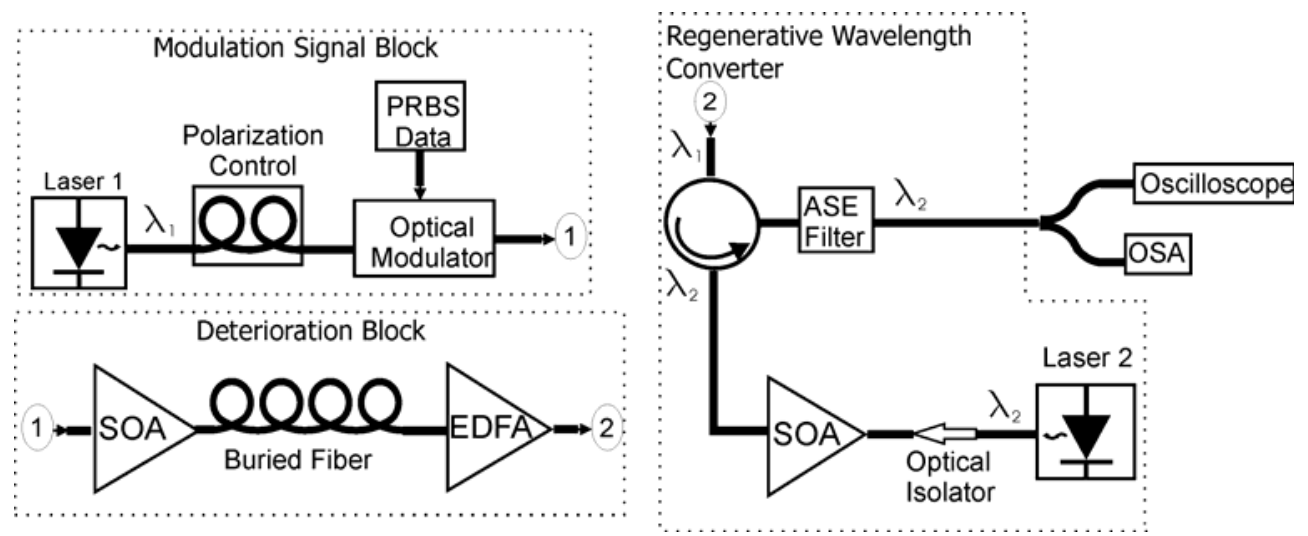

Fig. 1. All-optical 2R-regenerator and wavelength converter experimental setup.

The block for regeneration and wavelength conversion is the last one (Ribeiro et al., 2009a). In this block the modulated signal at $\lambda_{1}$ was converted to the wavelength of the laser $2\left(\lambda_{2}\right)$, occurring regeneration and wavelength conversion simultaneously. This $2 \mathrm{R}$-converter is a very simple device with just a laser CW (Continuous Wave), a non-linear SOA, an optical 
circulator and an optical isolator. These last two components are needed for operation in a counter-propagating mode of the wavelength converter based on XGM. The optical filter presented in this block was used to reduce the ASE noise added by the non-linear SOA, and to allow better eye diagrams visualization at the oscilloscope. If an oscilloscope with higher sensitivity was used, this optical filter might not be needed. In this way, this optical filter after the regenerator is not considered here as a regenerator component.

The SOA features are presented in Table 1. The non-linear commercial SOA was biased at $300 \mathrm{~mA}$ (near the maximum supported current of $400 \mathrm{~mA}$ ) to obtain the regenerative effects.

\begin{tabular}{|c|c|c|}
\hline Item & Test condition & Values \\
\hline Small signal gain & $\mathrm{I}=200 \mathrm{~mA}$ & $25-30 \mathrm{~dB}$ \\
\hline $\begin{array}{c}\text { Polarization dependent } \\
\text { saturated gain (PDG) }\end{array}$ & $\mathrm{I}=300 \mathrm{~mA}, \mathrm{P}_{\text {in }}>0 \mathrm{dBm}$ & $0.5-1 \mathrm{~dB}$ \\
\hline Saturated output power & $\mathrm{I}=200 \mathrm{~mA}$ & $6-8 \mathrm{dBm}$ \\
\hline Gain peak & $\mathrm{I}=200 \mathrm{~mA}$ & $1550-1570 \mathrm{~nm}$ \\
\hline Saturated gain recovery time & $\begin{array}{c}\mathrm{I}=300 \mathrm{~mA}, \mathrm{P}_{\mathrm{in}}>0 \mathrm{dBm}, \\
1555 \mathrm{~nm}\end{array}$ & $16-25 \mathrm{ps}$ \\
\hline 3 dB optical bandwidth & $\mathrm{I}=200 \mathrm{~mA}$ & $45 \mathrm{~nm}$ \\
\hline Active cavity length & & $2 \mathrm{~mm}$ \\
\hline Bias current & & up to $400 \mathrm{~mA}$ \\
\hline
\end{tabular}

Table 1. Parameters of the non-linear type encapsulated SOA.

In some deterioration cases, an optical attenuator was used before the oscilloscope to maintain the output signal power at the same level of the input signal, in order to carry out a bit reshaping comparison, excluding the regenerator gain.

Regenerator characterization was made for different parameter variations as for example: the optical power of lasers 1 and 2; bit rates; detuning; polarization angle of the input signal; and the extinction ratio (ER).

The different modulated input signal deteriorations cases are presented in the following subsections. Theses deteriorations were quantified by the signal Q-factor. This parameter is calculated by (Agrawal, 2002):

$$
Q=\frac{I_{1}-I_{0}}{\sigma_{1}+\sigma_{0}}
$$

In (1), $I_{1}$ and $I_{0}$ are the current level of the bits levels " 1 " and " 0 ", respectively; $\sigma_{1}$ and $\sigma_{0}$ are standard deviation of the level ' 1 ' and ' 0 ', respectively.

\subsection{Case "SOA"}

In this first deterioration case, another SOA was used to deteriorate the modulated input signal at $\lambda_{1}$. This SOA acted as a booster, amplifying and adding ASE noise. Depending on the power level of the input signal of this SOA, an overshoot related to the saturation of this device could happen. The 2R-converter performance is better for higher overshoot levels since this device totally removes the overshoot.

An optical band-pass filter was needed due to the higher level of ASE noise added to the signal. The modulated input signal Q-factor could be changed by varying the laser 1 power level and/or the bias current of the SOA used as a booster. 


\subsection{Case "LINK+SOA"}

In this deterioration case the modulated signal is degenerated by dispersion and attenuation of an 18-km standard buried fiber link of the KyaTera-Fapesp project (www.kyatera.fapesp.br). The fiber Corning SMF-28 Standard was used. The Table 2 shows some features of this fiber. Due to the attenuation, another SOA was used to amplify the signal in order to achieve the power level (at the entrance of the $2 \mathrm{R}$-converter) enough to reach regenerative effects, besides better visualization on the oscilloscope. The modulated input signal Q-factor could be changed in a similar way of the previous case.

\begin{tabular}{|c|c|}
\hline Item & Values \\
\hline Attenuation & $\leq 0.22 \mathrm{~dB} / \mathrm{km}$ \\
\hline Dispersion & 16 to $19 \mathrm{ps} / \mathrm{km}-\mathrm{nm}$ \\
\hline Effective Area & $80 \mu \mathrm{m}^{2}$ \\
\hline Numerical aperture & 0.14 \\
\hline Zero-dispersion wavelength & $1313 \mathrm{~nm}$ \\
\hline Polarization mode dispersion & $\leq 0.2 \mathrm{ps} / \mathrm{km}^{1 / 2}$ \\
\hline
\end{tabular}

Table 1. Fiber Corning SMF-28 features.

\subsection{Case "EDFA"}

In this deterioration case an EDFA was used to amplify the modulated input signal adding ASE noise. The higher ASE noise addition cause higher bit level (at both " 1 " and " 0 ") variance. The modulated input signal $\mathrm{Q}$-factor could be modified by varying the laser 1 power and/or EDFA pump laser power.

\subsection{Case "LINK+EDFA"}

This deterioration case is similar to the case "LINK+SOA". The 18-km standard buried fiber link of the KyaTera-Fapesp project was used again. The dispersion effects, attenuation, and ASE noise are the deterioration effects added to the modulated input signal.

\subsection{Case "SOA+LINK+EDFA"}

This deterioration case is the more complex case. It involves the three elements used to degenerate the modulated input signal. The overshoot appearance, the noise adding to bit levels " 1 " and " 0 " and high variance in those levels turn the modulated input signal presented in this case as the most deteriorated case. Optical filters were needed to reduce the ASE noise.

\section{2R-regenerator and wavelength converter working principle}

The quality of a $2 \mathrm{R}$ regenerator depends on its ability to suppress optical noise and to improve the extinction ratio. The ideal regenerative properties are provided by a system with a transfer function as close as possible to an ideal " $\mathrm{S}$ " like behavior. This refers to a characteristic function with the following properties: wide and flat dynamic range gain for bit levels " 1 " and " 0 " in order to suppress the noise, and a linear increasing curve which determines the discrimination between bit levels " 0 " and " 1 " (Simos et al., 2004).

The 2R-regenerator presented in this chapter has a characteristic function similar to the " $\mathrm{S}$ " like behavior. The response of the device here is similar, not equal to the " $\mathrm{S}$ ", since it 
presents gain compression only for the bit level " 1 ". This is due to the fact that just the power level of this bit (at the high level "1") can saturate the SOA gain. The compression of the level " 1 " is noticed in cases where an overshoot is presented. After the regenerator, the overshoot is removed because SOA gain is deeply saturated. In this way, the $2 \mathrm{R}$-converter works as a "low-pass filter", removing "higher frequencies" present in the overshoot. Therefore, saturated gain acts as a power equalizer for the fluctuation in the bit level " 1 " reducing the noise. On the other way, the bit level " 0 " has much less improvement than bit level " 1 ", since its low signal power level cannot saturate the SOA.

The 2R-converter of this chapter is based on XGM effect in SOAs, and this non-linear effect always degrades the extinction ratio due to the ASE noise added by the SOA. Consequently, there is no extinction ratio improvement. However, it will be showed in this chapter that the improvements caused by the $2 \mathrm{R}$-converter and quantified by Q-factor improvement can be higher than the ER degradation, at least for some cases.

The pattern dependence reduction caused by operating the $2 \mathrm{R}$-converter in its optimum optical input powers could improve both the format of the bit levels " 1 " and " 0 ". As it will be shown, there is an optimum relationship between the modulated input signal power (at $\lambda_{1}$ ) and the CW signal power (at $\lambda_{2}$ ), which should be maintained for different cases in order to kept the pattern dependence effects at the same level.

As mentioned before, the regeneration in the $2 \mathrm{R}$-converter occurs simultaneously with the wavelength conversion via XGM. The regenerative effects are associated with the wavelength conversion efficiency, and XGM is the simplest technique using SOA to implement wavelength conversion. In this process, a strong modulated input signal at $\lambda_{1}$ saturates the amplifier. A continuous wave pump signal at $\lambda_{2}$, injected simultaneously with the modulated signal, is modulated by the gain saturation while being concurrently amplified. The output signal, properly filtered, is a replica of the modulated input signal at a different wavelength with a phase inversion of $180^{\circ}$. In this way, methods to obtain the data without phase inversion are needed. One way is to use a proper software control. Commands given to the receiver force it to associate a bit " 1 " (in its input) to a bit " 0 " (in the original data). Another way is to use others devices like an additional SOA, but it will be enlarge the ER degradation. The exploiting of the phase changes provoked by the XGM effect using an optical filter could invert the output signal without ER degradation (Leuthold et al., 2003). Others forms are for example, the use of: delay interferometer (Liu et al., 2007) and quantum dots SOAs with narrow optical filters (Raz et al., 2008).

The eye diagrams obtained after the $2 \mathrm{R}$-converter presented in this chapter are inverted in phase. In this manner, the commentaries made before about the deterioration of the bit levels " 1 " and " 0 " refer to bit levels of modulated input signal. Otherwise, the improvement observed in the output signal is commented about the inverted bit levels, that is: for deterioration in the bit level " 1 " of the modulated input signal, the improvement is noted in the level " 0 " after the 2R-converter; and in a similar way for the deterioration in the level " 0 " of the modulated input signal.

The gain saturation of the SOA is related to the XGM wavelength conversion and to the gain compression. The last one is responsible for the noise reduction in the bit level " 1 " and for the overshoot elimination. The SOA gain must be deeply saturated to obtain the gain compression effects. This behavior can be noted in Figure 2. The eye diagram of the modulated input signal $\left(\lambda_{1}\right)$ deteriorated by the case "SOA" is presented in Figure 2(a). This eye diagram presents overshoot caused by the gain saturation of the SOA used as a booster that deteriorated the input signal. The eye diagram of the modulated signal $\left(\lambda_{1}\right)$ after the $2 \mathrm{R}-$ 
converter is presented in Figure 2(b). This much clear output signal was obtained for the regeneration case where it was observed a Q-factor improvement for the converted signal at $\lambda_{2}$ equal to 1.5. Due to the higher level power of the modulated and $\mathrm{CW}$ input signals, and the high SOA bias current $(300 \mathrm{~mA})$, the SOA in the 2R-converter presents a saturated gain. In this manner, the eye diagram for the output signal after the regenerator presents compressed eye. The overshoots and undershoots observed in the output signal might be associated to a self-phase modulation (SPM) and/or changes in the signal phase around the narrow band optical filter. These results of compressed eye diagrams were observed for other values of the modulated input signal power. Therefore it confirms that the gain of $\mathrm{SOA}$ used in the $2 \mathrm{R}$-converter is saturated, inducing the compression needed to the regenerative effects occurrence.

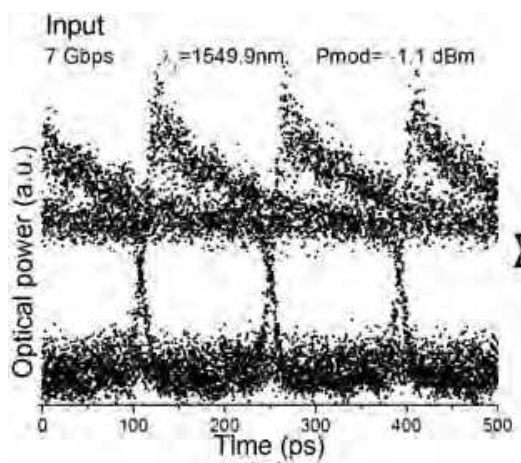

(a)

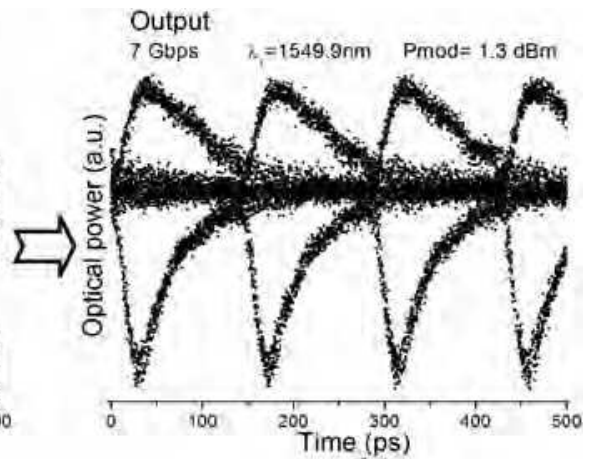

(b)

Fig. 2. Eye diagrams: (a) modulated input signal for the deterioration case "SOA"; (b) modulated signal after 2R-converter.

\section{Optical spectrum and signal to noise ratio}

The optical spectrum of the input and converted signals in those particular points of the experimental setup (Figure 1) are presented. The spectrums were obtained for the deterioration case "SOA" as an example, since the optical spectrum is similar to other deterioration cases. The optical signal to noise ratio (OSNR) was calculated as well as the eye diagrams were obtained corresponding to the optical spectrums illustrated in Figure 3.

The case of Figure 3 employed a wavelength conversion from 1550 to $1551 \mathrm{~nm}$ with a modulation rate of $7 \mathrm{Gbps}$ NRZ. The Figure 3(a) shows the modulated input signal without deterioration and an eye diagram without distortions. A Q-factor of 9 and OSNR of $55.28 \mathrm{~dB}$ are observed in this case. Then this modulation input signal is inserted into the SOA used as a booster. The gain of this SOA is saturated due to the power level of the input signal of -2 $\mathrm{dBm}$ and a bias current of $130 \mathrm{~mA}$ (higher than current threshold). Therefore the eye diagrams presented in Figure 3(b) are obtained with much noise in the bit levels " 1 " and " 0 " as well as overshoots. After the SOA used as a booster, the Q-factor decrease to 4.3 as well as the OSNR to $37.6 \mathrm{~dB}$. In this manner, this SOA presented a noise figure of $18.3 \mathrm{~dB}$ caused by deeply saturated gain and by extra noise added to the signal. An optical band-pass filter is needed to filter the ASE noise added. Consequently, the signal in Figure 3(c) appeared filtered with an improvement in the Q-factor to 4.8. 


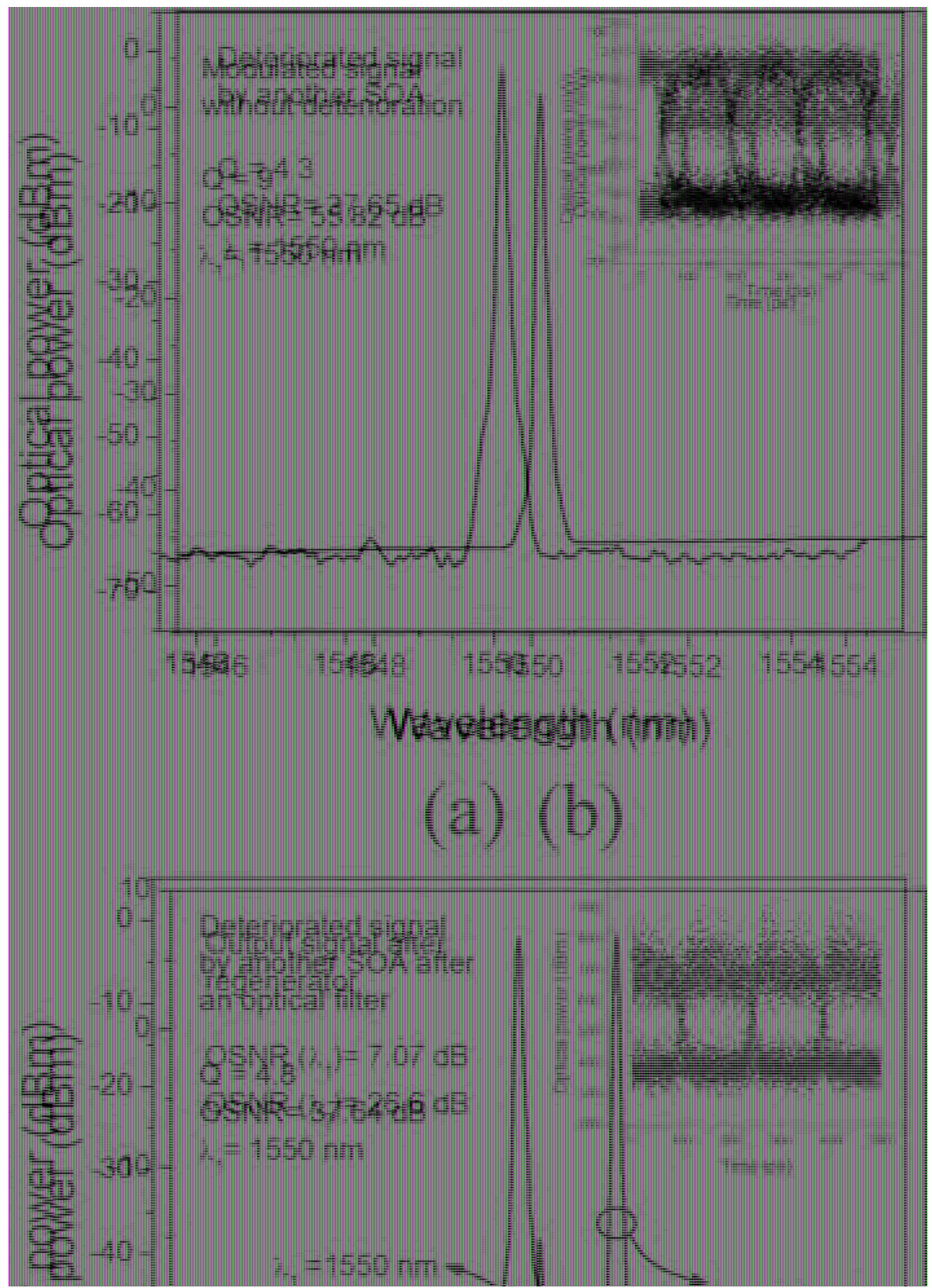

Fig. 3. Optical spectrums and eye diagrams of the deterioration case "SOA": (a) modulated signal without deterioration; (b) modulated signal deteriorated by another SOA; (c) modulated signal deteriorated by another SOA and filtered; (d) output signal after the 2Rconverter; and (e) regenerated signal and filtered. 
The optical spectrum after the 2R-converter is presented in Figure 3(d). The input signal initially at $1550 \mathrm{~nm}\left(\lambda_{1}\right)$ is still present but with an OSNR of $7 \mathrm{~dB}$. According to the experimental setup of Figure 1, the original signal at $\lambda_{1}$ should not be present because the conversion scheme is a counter-propagating mode with an optical isolator that should eliminate this original signal. Nevertheless, the original signal presence after the $2 \mathrm{R}$ converter could be explained by possible internal reflections in the optical isolator and in the SOA used to regenerate the signal. The regenerated and converted signal at $1551 \mathrm{~nm}\left(\lambda_{2}\right)$ of Figure 3(d) presents an OSNR of $26.6 \mathrm{~dB}$, i. e., the SOA used in the regenerator presented a noise figure of $11 \mathrm{~dB}$. As the same case mentioned before, this noise figure higher than commons values $(7 \mathrm{a} 8 \mathrm{~dB})$ could be justified by the presence of both the modulated input signal power $(-2 \mathrm{dBm})$ and the $\mathrm{CW}$ signal power $(-6 \mathrm{dBm})$ as well as higher bias current (300 $\mathrm{mA}$ ) which saturated the SOA gain, adding a lot of noise to the signal. Eye diagram is not illustrated in Figure 3(d) due to the high power level of the output signal $(7 \mathrm{dBm})$. Indeed, the bit level "1" of the output signal eye diagram was in the upper part of the oscilloscope scale limit, not allowing the acquisition of points by the software Labview (using GPIB port). Despite this limitation, a reduction of the noise in bit levels " 1 " and " 0 " and an overshoot elimination were noted in the eye diagrams (quantified by Q-factor improvement from 4.8 to 7.2). These results evince that the original signal still present at $\lambda_{1}$ does not decrease the regenerative effects.

The regenerated and converted signal at $1551 \mathrm{~nm}\left(\lambda_{2}\right)$ after an optical narrow filter is illustrated in Figure 3(e). The optical filter allows an OSNR improvement to $63.2 \mathrm{~dB}$. The eye diagram observed in this figure presents improvements already mentioned in the previous case. These improvements increase the Q-factor to 7.5. Through calculating the Q-factor variation from the modulated input signal, an improvement of 2.7 can be observed.

For the cases presented in this section and in Figure 3, the results after the regenerator were not attenuated to guarantee the same power level of the modulated input signal. This was made to allow the observation of the $2 \mathrm{R}$-converter performance as a whole, analyzing the reamplification and reshaping.

The calculation of the OSNR was made following application notes published by the manufacturer of the optical spectrum analyzer used here. Therefore the optical spectrums presented in Figure 3 are just illustrations since the accurate OSNR calculations need an operation of the optical spectrum analyzer with higher resolution and smaller span.

The optical spectrums and the OSNR were presented just for the deterioration case "SOA". The optical spectrums for the others deterioration cases are similar, presenting differences in the optical power values. In relation to the OSNR calculation, the values obtained for the other deterioration cases are very close, with variations due to: the optical signal power used; bias current of the SOA; and the pump laser power used in the EDFA. A study of the ER degradation will be presented in following sections in order to analyse the signal degradation after the 2R-converter that was caused by the ASE noise addition of the SOA. This study will help to understand how the noise degenerate the signal for different cases, associating these results with the OSNR deterioration. In this manner, it will be possible to estimate the OSNR behavior for the different deterioration cases not presented in this section.

\section{Re-amplification}

The $2 \mathrm{R}$-converter presented in this chapter provides re-amplification and bit reshape. Thus, the first improvement caused by this regenerator is the signal re-amplification that will be 
present in this section. The deterioration case "SOA" is used to illustrate the optical gain originated by the 2R-converter. A wavelength conversion from $1550 \mathrm{~nm}\left(\lambda_{1}\right)$ to $1551 \mathrm{~nm}\left(\lambda_{2}\right)$ with a bit rate of $10.3 \mathrm{Gbps}$ was used. The optical gain was calculated as the difference between the output signal power at $\lambda_{2}$ and input modulated signal power at $\lambda_{1}$. The results are presented in Figure 4.

The optical gain versus CW signal power at $\lambda_{2}$ is illustrated in Figure 4(a). A gain increase with the CW signal power can be noted. This happens because the output signal was kept at $\lambda_{2}$. Thus, by increasing the CW signal power, the output signal power at $\lambda_{2}$ increases too. Since the optical gain was calculated as a function of the modulated input signal power, which is fixed for each curve in Figure 4 (a), the optical gain increases linearly with the CW signal power. In Figure 4(b), an optical gain decreasing with the modulated signal power increasing is noted, presenting higher optical gain values for the modulated signal power around $-7.5 \mathrm{dBm}$. This result is associated with the SOA gain saturation. Some Q-factor improvements (figured by $\Delta \mathrm{Q}$ ) are showed in Figure $4(\mathrm{a})$ and (b) just to illustrate the dependence of this parameter with the power relation, which will be commented in other section. Here, $\Delta \mathrm{Q}$ is defined as the difference between the Q-factor of converter signal at $\lambda_{2}$ and Q-factor of the modulated input signal at $\lambda_{1}$.

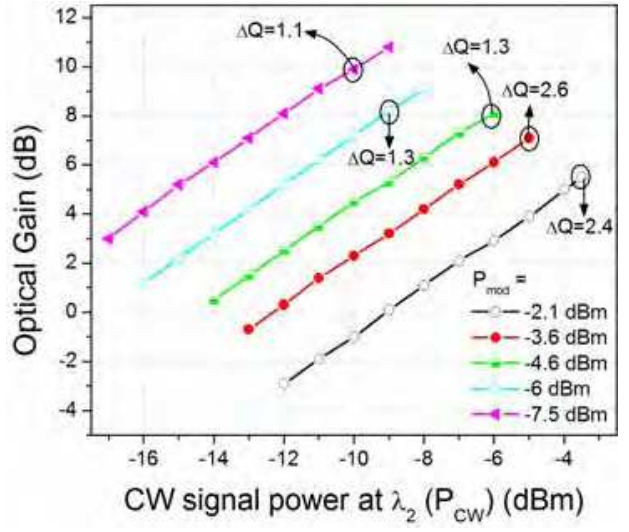

(a)

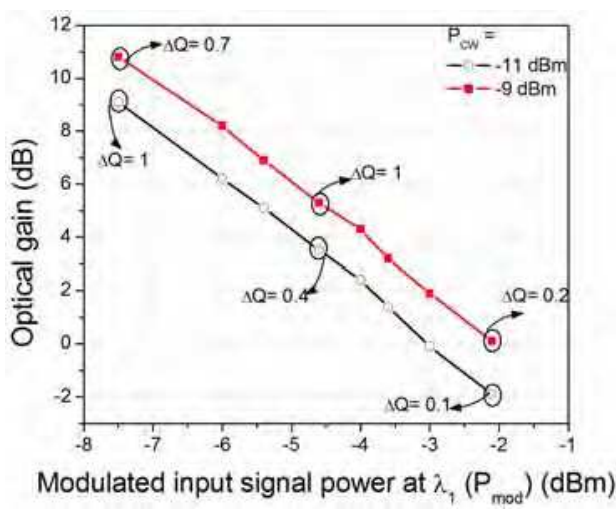

(b)

Fig. 4. 2R-converter optical gain of the deteriorated case "SOA": (a) optical gain versus CW signal power for different input modulated signal powers; (b) optical gain versus input modulated signal power for different $\mathrm{CW}$ signal power.

The $2 \mathrm{R}$-converter presented an optical gain varying from -3 to $12 \mathrm{~dB}$. In most of the cases, the better values of $Q$-factor improvement occurred for higher optical gain values. In this manner, it is clear that the $2 \mathrm{R}$-converter is capable to re-amplify the signal, presenting optical gain up to $12 \mathrm{~dB}$, together with the bit reshape quantified by the Q-factor improvement.

These results of optical gain presented here are proper of the SOA and can be associated to the input modulated signal power and CW signal power. In this way, if the same values of the input optical powers in Figure $4(\mathrm{a})$ and (b) is used for the others deterioration cases, the results should be similar to the ones presented here. 


\section{Eye diagrams}

The eye diagrams obtained from the oscilloscope clarify the improvements obtained by the $2 \mathrm{R}$-converter. In this section, some eye diagrams of the different deteriorated cases are presented to illustrate the improvements of the bit shape. An up-conversion (1550 to 1551 $\mathrm{nm}$ ) was employed to obtain the eye diagrams. This type of conversion causes higher ER deterioration, but in other way it also provides higher SOA gain saturation, which contributes to a better signal regeneration. Therefore, all the eye diagrams presented in this section as well as most of the results presented in this chapter were obtained from upconversion. In addition, it is valid to comment that the output eye diagrams are inverted in relation to input signal.

Initially, input and output eye diagrams and the respective Q-factors for the bit rate of 10.3 Gbps NRZ are illustrated in Figure 5, where the output signal was not attenuated to the same level of the input modulated signal power. Therefore the illustrated eye diagrams present two regeneration effects: re-amplification and reshaping. An arbitrary unit for the optical power was considered to allow the comparison between input and output eye diagrams in same proportion.

Two deterioration cases are studied. The first case is "LINK+SOA", which presented a medium quality input signal (Figure 5(a)) with Q-factor of 5.7, presenting intense pulse distortion due to the intrinsic dispersion caused by the $18-\mathrm{km}$ standard buried fiber link (Ribeiro et al., 2009a). The dispersion effect could be noted by the triangular form of the pulse. In Figure 5(b), the regenerated output signal presents a higher eye opening, a reduction of the overshoots, and of the bit level (at both " 1 " and " 0 ") variance, facts quantified by the $\mathrm{Q}$-factor increasing to $\mathrm{Q}=10$.
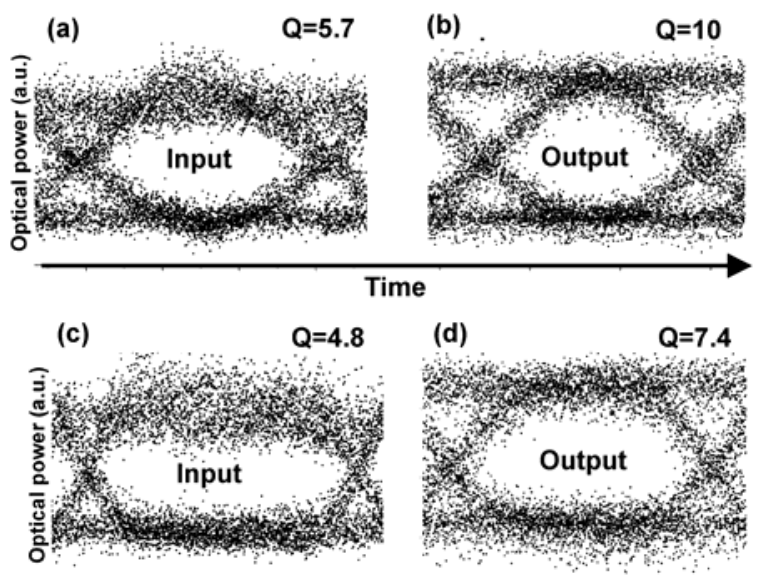

Fig. 5. Eye diagrams (NRZ, $10.3 \mathrm{Gbps}$ ): (a) case "LINK+SOA" input signal with $\mathrm{Q}=5.7$; (b) output signal with $\mathrm{Q}=10$; (c) case "SOA" input signal with $\mathrm{Q}=4.8$; (d) output signal with $\mathrm{Q}=7.4$ (adapted from Ribeiro et al., 2009a).

The second deterioration case is "SOA" (Ribeiro et al., 2009a). In Figure 5(c), the eye diagram presents low quality $(\mathrm{Q}=4.8)$ due to the pattern dependence effect, overshoots, and the great amount of noise added to both bit levels by the SOA used as a booster. As the case mentioned before, an improvement in the eye opening can be observed as well as an 
overshoot elimination is noted by the small variance of the bit level " 0 " of the regenerated signal. In addition, the bit levels " 1 " and " 0 " of the regenerated signal present lower width (reduction of the bit level variance) if be compared to the inverted bit level of the input signal. These improvements are quantified by the increasing of the Q-factor to 7.4.

Eye diagrams for bit rate of $7 \mathrm{Gbps}$ for the same cases mentioned before are presented in Figure 6. An important difference is that in Figure 6 the output signal is attenuated to guarantee the same level of modulated input signal power. In this manner, the unit of $\mu \mathrm{W}$ could be used. This study of output signal attenuated analyzes just the improvement provoked by the bit reshaping.

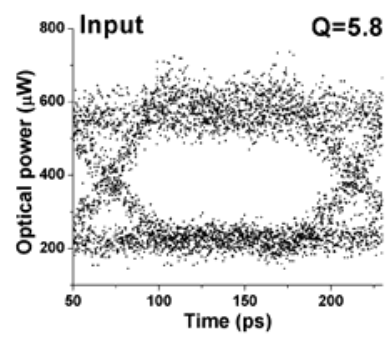

(a)

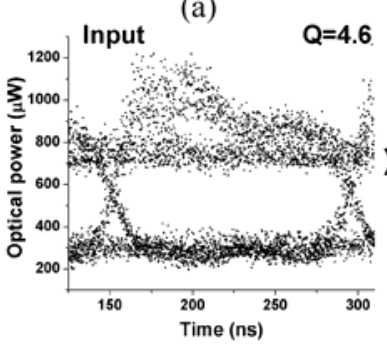

(c)

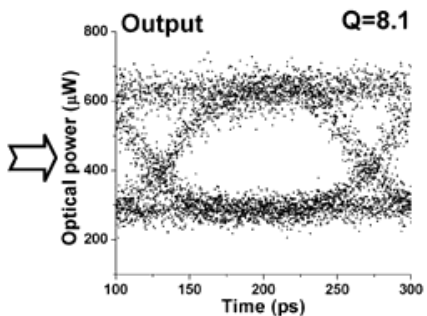

(b)

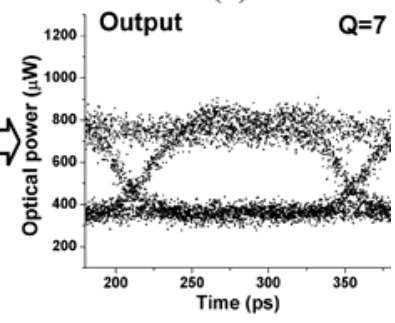

(d)

Fig. 6. Eye diagrams (NRZ, 7 Gbps): (a) case "LINK+SOA" input signal with $Q=5.8$; (b) output signal with $\mathrm{Q}=8.1$; (c) case "SOA" input signal with $\mathrm{Q}=4.6$; (d) output signal with $\mathrm{Q}=7$.

Despite the output signal attenuation, the behavior is similar to the cases mentioned in Figure 5. In case "LINK+SOA", the deterioration effects presented in the input signal of Figure 6(a) are the same presented in previous figure, as well as the improvements in the output signal (Figure 6(b)). These similarities are quantified by the Q-factors 5.8 and 8.1 for the input and output signal respectively. A decreasing of the Q-factor improvement can be noted by comparing to the previous case. This result is due to the attenuation of the output signal. Besides, this is another situation where the modulated and CW signals powers are different from the cases of Figure 5. The eye diagrams illustrations are used to observe the improvement caused by the $2 \mathrm{R}$-converter, comparing the input and output signal in each case, and not to make comparisons between the different deterioration cases where different parameters are used.

The deterioration case "SOA" is presented in Figure 6(c). The input signal presented a higher overshoot as well as deterioration caused by the pattern dependence effect and ASE noise added by the SOA used as a booster. The output signal presents overshoot elimination and lower variance of both bit levels. The improvements are quantified by the Q-factor increasing from 4.6 to 7 . 
The others deterioration cases like "LINK+EDFA" and "EDFA" are illustrated in Figure 7. These eye diagrams were obtained for a bit rate of 7 Gbps NRZ. Besides, the output signal was attenuated to guarantee the same level of the modulated input signal power. In the case "LINK+EDFA", the input signal illustrated in Figure 7(a) presents a high amount of ASE noise added by the EDFA, and deterioration caused by the dispersion of the buried fiber link. The bit levels of " 1 " and " 0 " present a large width, i.e., high variance. In the output signal (Figure $7(b)$ ) the increasing of the eye opening is noticeable. It is caused by the decrease of the noise present in bit levels " 1 " and " 0 ", visualized by the variance reduction of these levels. A higher noise reduction is noted to the bit level " 0 " of the regenerated signal. The Q-factor was increased from 4.5 to 6.2.

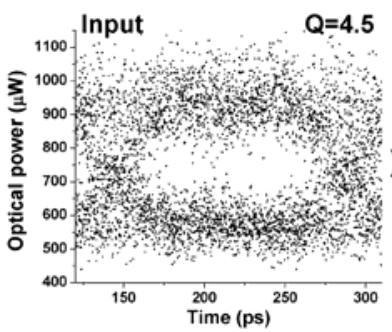

(a)

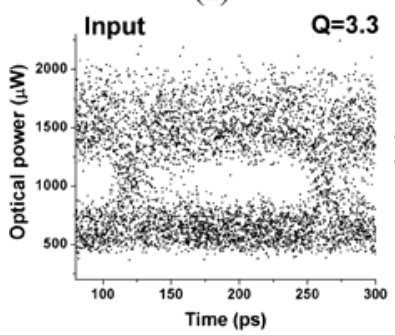

(c)

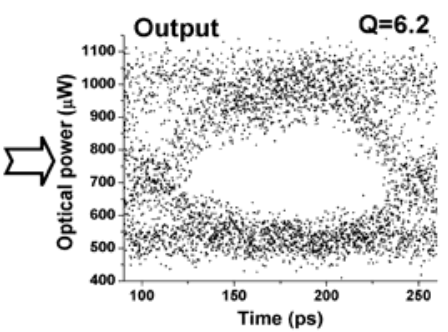

(b)

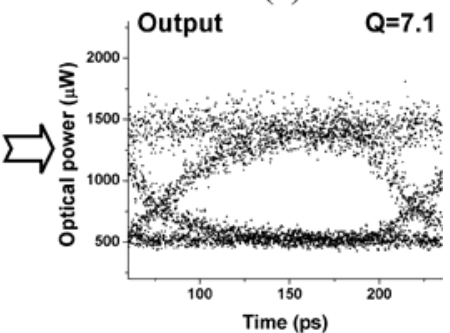

(d)

Fig. 7. Eye diagrams (NRZ, 7 Gbps): (a) case "LINK+EDFA" input signal with $Q=4.5$; (b) output signal with $\mathrm{Q}=6.2$; (c) case "EDFA" input signal with $\mathrm{Q}=3.3$; (d) output signal with $\mathrm{Q}=7.1$.

The deterioration case "EDFA" is illustrated in Figure 7(c). A great amount of ASE noise deteriorating the input signal with a low eye opening can be noted. This higher deterioration presented in the input signal is quantified by the low Q-factor of 3.3. In Figure $7(\mathrm{~d})$, the improvement caused by the $2 \mathrm{R}$-conveter can be observed. Due to the SOA gain saturation, the noise is reduced in both bit levels " 1 " and " 0 ". In the last one bit level, a lower variance can be noted. With the ASE noise reduction, the eye opening increase as well as the Q-factor to 7.1.

The last deterioration case illustrated involves all the degeneration effects: "SOA+LINK+EDFA". For this last case, a bit rate of 7 Gbps NRZ as well as output signal attenuation are used (Ribeiro et al., 2009a). The Figure 8(a) illustrates the input signal which presents a higher overshoot caused by the SOA used as a booster. Besides, the input signal presents higher variance in both bit levels provoked by the ASE noise addition by the SOA and EDFA. The input signal also presents a bit enlargement caused by the intrinsic 
dispersion of the buried fiber link. The output signal illustrated in Figure 8(b) presents a noise reduction in both bit levels. The overshoot was reduced as well as the fluctuations presented in the bit level " 1 ". Nevertheless, the output signal presents a lower difference between the bit levels " 0 " and " 1 ", i. e., lower extinction ratio (ER). The improvement observed in Q-factor was from 5.3 to 8.6.

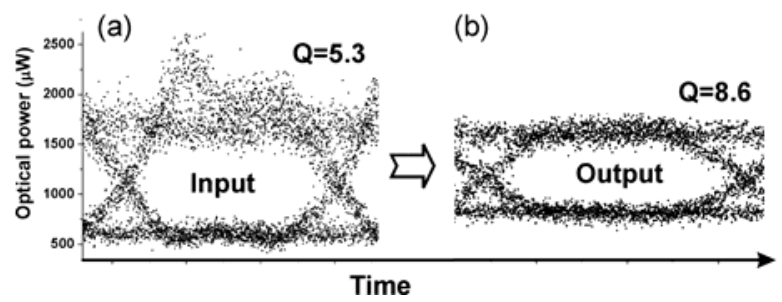

Fig. 8. Eye diagrams (NRZ, 7 Gbps): (a) case "SOA+LINK+EDFA" input signal with $Q=5.3$; (b) output signal with $Q=8.6$ (adapted from Ribeiro et al., 2009a).

Due to the availability of equipments, the same bit rate could not be used for all the cases studied. In this manner, the use of modulation rate higher than 7 Gbps NRZ was used just for some measurements, but for most cases the characterization was limited to 7 Gbps.

By comparing the eye diagram of the output signal with the input, a reduction of cross point level between " 0 " and " 1 " can usually be noted. This reduction is more pronounced for cases where the output signal is attenuated. The ER degeneration is the main reason for this cross point level reduction. Another reason is the SOA gain recovery time. The rising time of the bit level " 1 " is slower than the falling time, decreasing the cross-point level.

The modulation RZ (Return to zero) was used in the 2R-converter characterization either. The Figure 9 presents the eye diagrams for R1 modulation that correspond to inverted RZ. A wavelength conversion from 1550 to $1551 \mathrm{~nm}$ was used without attenuation in the output signal. Figure 9(a) illustrates the input signal for the deterioration case "LINK+SOA". The pulse presents a triangular shape due to the buried fiber link. A decreasing in the variance of the bit level " 1 " of the input signal can be noted in Figure 9(b). An estimation of the regenerative effects was done using the variance of both bits levels. An improvement of $51 \%$ was obtained for this deterioration case.

The deterioration case "SOA" is illustrated in Figure 9(c), presenting pulse shape more rectangular and more ASE noise in the bit level "1". The output signal (Figure 9(d)) presents narrower pulses due to the gain response time of the SOA. Besides, there is a reduction in the bit level " 1 " variance. As the previous case, an estimate was calculated to eye opening improvement being obtained $44 \%$.

These results proved that the $2 \mathrm{R}$-converter is capable to regenerate RZ signals. Nevertheless, the results present in this chapter use just NRZ modulation since this modulation type is more complex. Furthermore the Q-factor used to quantify the regenerative effects is just provided by the oscilloscope for the NRZ modulation type.

The overshoot elimination presented for the cases in which another SOA was used to amplify the modulated input signal is a good feature of the $2 \mathrm{R}$-converter. In optical systems, the overshoot could be added to the signal from different forms, one of them is the use of the PISIC technique used to increase the speed of the electro-optical switching using SOAs (Gallep \& Conforti, 2002) (Ribeiro et al., 2009b). 


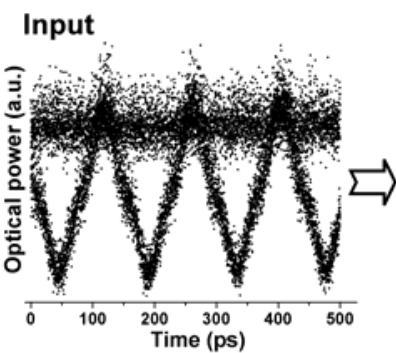

(a)

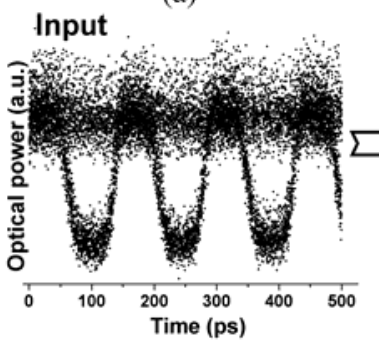

(c)

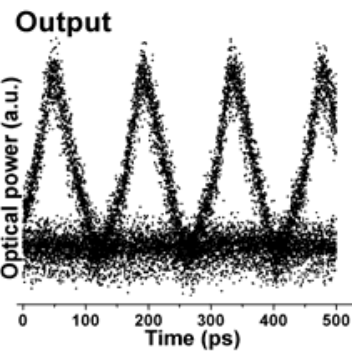

(b)

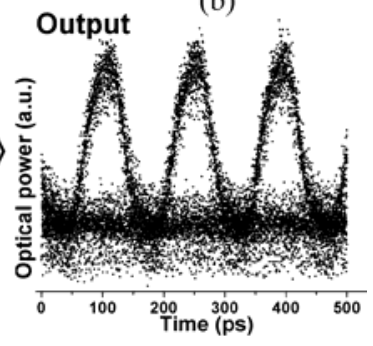

(d)

Fig. 9. Eye diagrams (R1, 7 Gbps): (a) case "LINK+SOA" input signal; (b) output signal; (c) case "SOA" input signal; (d) output signal.

The overshoots presented in the deterioration case "SOA", "LINK+SOA", and "SOA+LINK+EDFA" are eliminated by the 2R-converter as could be observed in Figure 5, 6, and 8 . This elimination occurs due to the saturation effects of the SOA gain. The SOA does not maintain the gain level for those higher power values present in the overshoots. In this manner, the overshoot is not transferred to the $\mathrm{CW}$ signal by the wavelength conversion.

Therefore, the 2R-converter is a possible solution to eliminate the overshoot of an optical signal. Despite the overshoot elimination, the signal after the regenerator will present ER decreasing, as it will be shown in future sections. Thus, the analysis if the overshoot elimination can compensates for the ER degeneration is necessary.

\section{Optical polarization}

The input light polarization dependence of the wavelength conversion is very important since polarization is an unpredictable factor in real optical systems and an automatic polarization controller can be expensive. The SOA used in the $2 \mathrm{R}$-converter presents a polarization dependent saturated gain (PDG) of less than $1 \mathrm{~dB}$. Studies of the input polarization angle influence in the $2 \mathrm{R}$-converter performance were done to confirm this value.

By adjusting de polarization controller, different polarization angles of the modulated input signal were obtained to analyse the Q-factor and gain variation. The Figure 10 presents eye diagrams of different polarization angles for the deterioration case "SOA". The modulated input signal changes very little for each polarization angle. In this manner, the eye diagram presented as input is representing all the input eye diagrams. The eye diagrams following in the time scale illustrate the variations noted in the output signal for some input polarization angles. The regenerative effects are presented in all the output signal with overshoot elimination and the reduction of the bit levels " 1 " and " 0 " variance. 


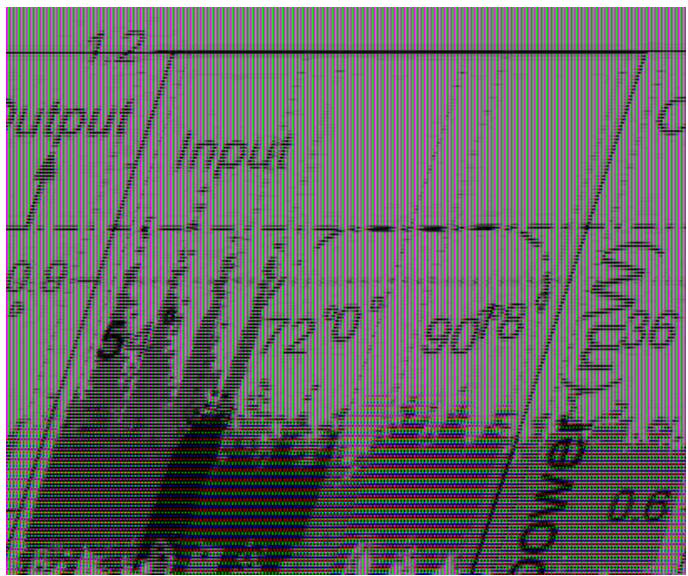

Fig. 10. Eye diagrams for different polarization angles of the input signal for the deterioration case "SOA".

Observing the eye diagrams for different polarization angles of the input signal, regenerative and power variations could be noted. These variations can be better observed in Figure 11 (a) where the optical gain varying from 0.6 to $1.5 \mathrm{~dB}$ is observed. In addition, it was observed that for this deterioration case, the $\mathrm{Q}$-factor improvement $(\Delta \mathrm{Q})$ varied from 0.7 to 1.9 .

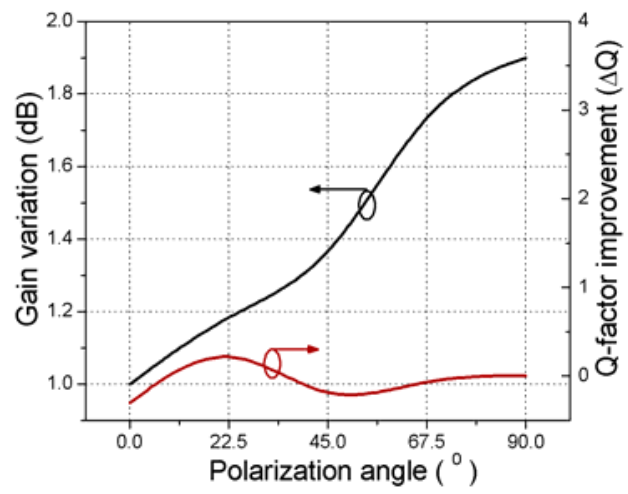

(a)

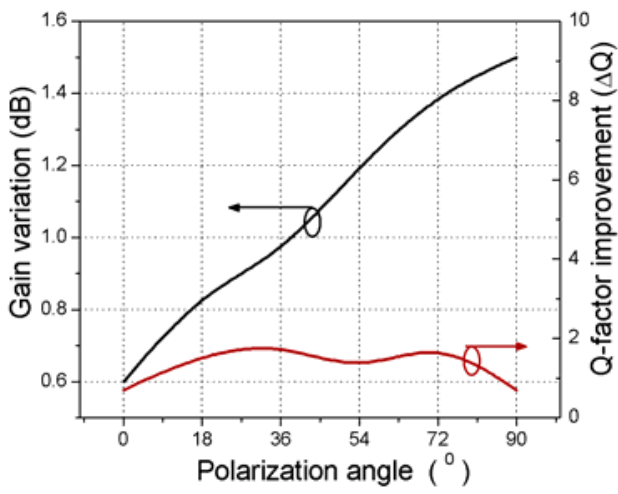

(b)

Fig. 11. Gain and Q-factor improvement variation as function of different input polarization angles for the deterioration case: (a) "SOA" and (b) "LINK+SOA" (adapted from Ribeiro et al., 2009a).

A study of the case "LINK+SOA" was done in a similar manner, obtaining the results presented in Figure 11(b). In this case, a gain variation of $0.9 \mathrm{~dB}$ and a $\mathrm{Q}$-factor improvement variation of 0.9 were obtained.

In general, the $2 \mathrm{R}$-converter presented low dependence with the input signal polarization, fact proved by the results in Figure 11, with a gain variation of less than $1 \mathrm{~dB}$. This behavior is explained by the use of a SOA with low PDG. Thus, the 2R-converter has this advantage 
of low polarization dependence, with a practical effect in real systems where the fiber income signal polarization is not known or cannot be controlled.

\section{Input optical power: the modulated and the $\mathrm{CW}$ signals}

The power levels of the modulated $\left(\lambda_{1}\right)$ and CW $\left(\lambda_{2}\right)$ signals are important to achieve the SOA gain saturation used in the $2 \mathrm{R}$-converter, necessary for regeneration and conversion by XGM effect. Moreover, the power levels of these input signals affect the power level of the output signal and the quality of the input signal. A study of the Q-factor improvement for different power level pairs of $\mathrm{CW}$ and modulated channels, for each different case of deterioration was done. For the cases presented in this section, up-conversions were performed from 1550 to $1551 \mathrm{~nm}$ at a rate of $7 \mathrm{Gbps}$, with attenuation of the output power to guarantee the same level in relation to the modulated input signal power.

In the Figure 12(a) (case "SOA") it is noted that there is a Q-factor improvement increasing while the $C W$ signal power increases, with a maximum $\Delta \mathrm{Q}$ value for a power value of the $\mathrm{CW}$ signal above from 2 to $3 \mathrm{~dB}$, compared to the modulated signal input power. After this maximum, there is a decrease of $\Delta \mathrm{Q}$ with the $\mathrm{CW}$ signal power increasing. In the case of the $-9 \mathrm{dBm}$ modulated signal power, there is a different behavior with two $\Delta \mathrm{Q}$ value peaks, which occur for values of $\mathrm{CW}$ signal power equal to or greater than $1 \mathrm{~dB}$ in relation to the modulated signal power. However, the decrease after these peaks is maintained.

The relation between power and Q-factor improvement can be understood considering the cases of $-6.7 \mathrm{dBm}$ and $-7.6 \mathrm{dBm}$ of modulated signal power. In these cases, for levels of $\mathrm{CW}$ signal power lower than the modulated input signal, the SOA does not reach the desired saturation to attain greater conversion efficiency and regenerative effects. Therefore, higher $\Delta \mathrm{Q}$ values do not exist. However, for $\mathrm{CW}$ signal power values above from 2 to $3 \mathrm{~dB}$, the ideal saturation is accomplished. Moreover, the power level of the output signal at $\lambda_{2}$ becomes more influenced by this $\mathrm{CW}$ signal power. From this value of $\mathrm{CW}$ power, the SOA gain saturation becomes very intense due to the higher power injected in this device, without efficiency in the XGM conversion, leading to a $\triangle Q$ decreasing.

Another factor that influences the behavior of these curves is the input signal quality, which is dependent of the modulated signal power. For example, the curve of the $-9 \mathrm{dBm}$ modulated signal power has a different behavior and lower $\Delta \mathrm{Q}$ values, because it has the highest Q-factor initial value, which is 6 while the other cases vary from 4.2 to 5 . This curve has the highest Q-factor even with the lowest power level, because the signal has low amplification from the SOA used as a boster, adding less noise and overshoot. Thus, the $\Delta \mathrm{Q}$ improvement is small because it has a large input $\mathrm{Q}$ value. A $\mathrm{Q}$-factor improvement up to 3.3 for a total input power $\left(\mathrm{P}_{\mathrm{mod}}+\mathrm{P}_{\mathrm{CW}}\right)$ of $-9.3 \mathrm{dBm}$ was observed. Besides that, it was obtained a Q-factor improvement for input power values from $-15.8 \mathrm{dBm}$ to $-1.68 \mathrm{dBm}$.

The case "LINK+SOA" is shown in the Figure 12(b), where is possible to observe a behavior like the case "SOA", with maximums of $\Delta \mathrm{Q}$ for the $\mathrm{CW}$ signal power above 1 to $2.5 \mathrm{~dB}$ compared to the modulated signal input power. The justification for this behavior is also associated with the SOA gain saturation. For this case, lower $\Delta \mathrm{Q}$ values were obtained. However, it was observed a Q-factor improvement for values of total input power from -19 $\mathrm{dBm}$ to $-6 \mathrm{dBm}$. Since there is link attenuation, the power values of the modulated signal could be extended to small power values $(-12.2 \mathrm{dBm})$. 


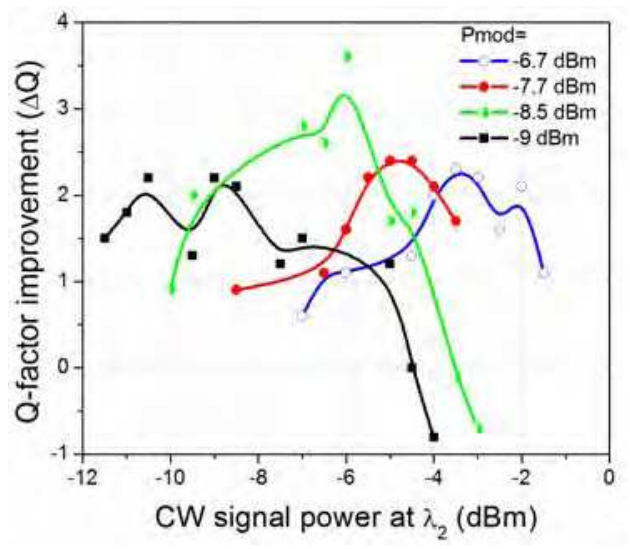

(a)

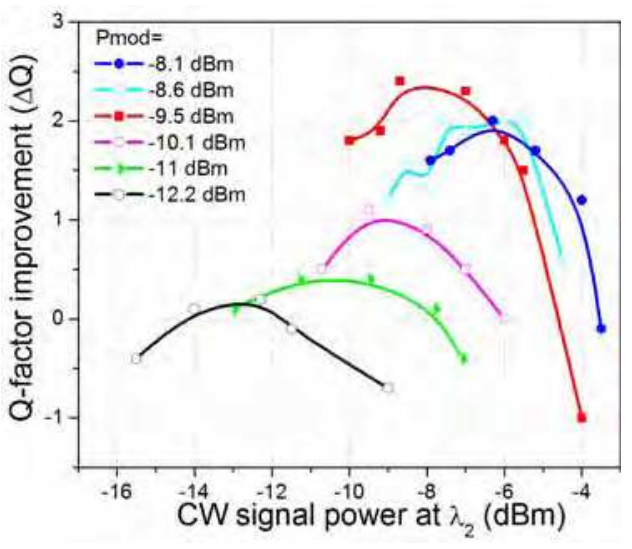

(b)

Fig. 12. Q-factor improvement versus input optical power (modulated and $\mathrm{CW}$ ) for the deterioration cases: (a) "SOA" and (b) "LINK+SOA".

The Figure 13(a) (case "EDFA") shows the Q-factor improvement as a function of CW signal power for three cases of modulated signal input power. It reveals a similar behavior, but with less $\Delta \mathrm{Q}$ variation, since the scale is represented from 3.2 to 4.8 units. Moreover, there are more oscillations between $\Delta \mathrm{Q}$ minimums and maximums. This behavior may be partly explained by the utilized scale, since these variations between minimums and maximums are actually just from 0.2 to 0.4 units. Another reason may be inaccuracies when the Q-factor values were obtained directly from the oscilloscope.

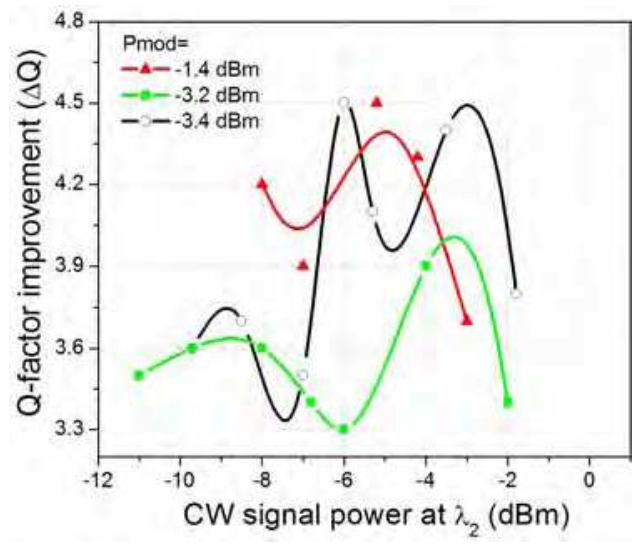

(a)

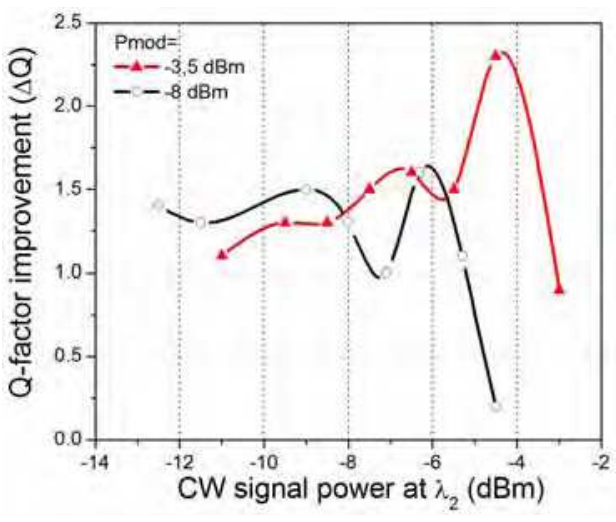

(b)

Fig. 13. Q-factor improvement versus input optical power (modulated and CW) for the deterioration cases: (a) "EDFA" and (b) "LINK+EDFA".

The $\Delta \mathrm{Q}$ maximums occurred for values of the $\mathrm{CW}$ signal power from 0.8 to $3.6 \mathrm{~dB}$ below the values of the modulated signal input power. This occurs since the power levels of the modulated signal already have high values; therefore the CW signal powers cannot assume 
values higher than the power levels of the modulated signal, otherwise more SOA gain saturation will occur with consequent $\Delta \mathrm{Q}$ reduction. For this case, it was attained a $\mathrm{Q}$-factor improvements for total input power values from $-5.8 \mathrm{dBm}$ to $2 \mathrm{dBm}$, with a $\Delta \mathrm{Q}$ maximum of 4.8 .

The deterioration case "LINK+EDFA" is illustrated in the Figure 13(b), showing power values of the modulated input signal lower than the previous case, owing to attenuation caused by the buried fiber link. These curves also present oscillations as a result of inaccuracies. However, disregarding the minimum $\Delta \mathrm{Q}$ values, the behavior is similar to that observed previously. The $\Delta \mathrm{Q}$ maximums have different behavior for the two power values of the modulated input signal. For the case of $-3.5 \mathrm{dBm}$, the $\Delta \mathrm{Q}$ maximum occurs for the $\mathrm{CW}$ power level of $-4.5 \mathrm{dBm}$. Nevertheless, for the case of the modulated signal input power of $8 \mathrm{dBm}$, the $\Delta \mathrm{Q}$ maximum occurs for power values of the $\mathrm{CW}$ signal of $-6 \mathrm{dBm}$. These results are also related to the SOA gain saturation. It was achieved a Q-factor improvements for values of total input power from $-15.6 \mathrm{dBm}$ to $-1.1 \mathrm{dBm}$, with $\Delta \mathrm{Q}$ maximum of 2.3.

The last deterioration case ("SOA+LINK+EDFA") is presented in the Figure 14. It is possible to note a behavior similar to the others, presenting a $\triangle \mathrm{Q}$ increasing with the $\mathrm{CW}$ signal power up to a maximum, and then a decrease for the higher values of $C W$ signal power. The $\Delta \mathrm{Q}$ maximums occur for power values of the $\mathrm{CW}$ signal below from 0.5 to $1.5 \mathrm{~dB}$ compared to the modulated input signal. It was obtained $Q$-factor improvements for values of total input power from $-12 \mathrm{dBm}$ to $1.76 \mathrm{dBm}$, with $\Delta \mathrm{Q}$ maximum of 4 .

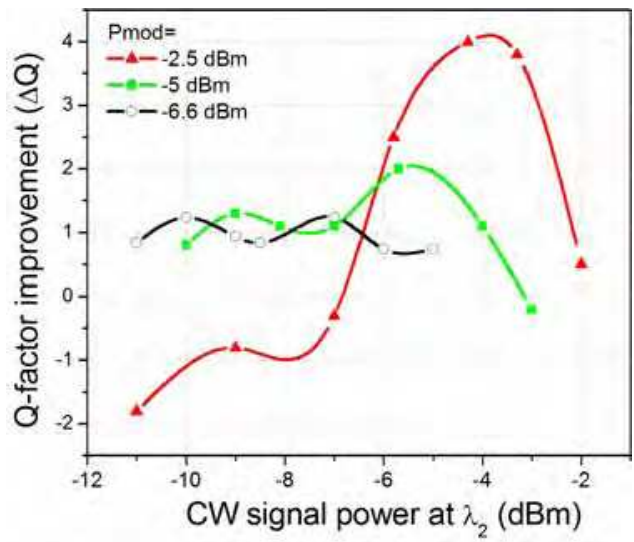

Fig. 14. Q-factor improvement versus input optical power (modulated and CW) for the deterioration case "SOA+LINK+EDFA".

From the results presented in the previous figures, it is possible to observe that there is an optimum relation between the powers of the modulated input signal and the CW signal. This relation is associated to the SOA saturation levels, varying for each deterioration case. In addition, it is possible to note that the higher values of Q-factor improvements occur for the case "EDFA", where the power levels of the modulated signal are the highest with the lowest Q-factor values for the modulated input signal, because the EDFA provides large amplification. As a result, small values of initial Q-factor allow more possibility for improvement. In the cases where another SOA is used to amplify the signal, causing overshoot, high $\Delta \mathrm{Q}$ values were also obtained. As seen in the eye diagrams presented in the 
Section 6, the 2R-converter eliminates overshoot, consequently providing more improvements in the Q-factor.

Analyzing the use of the 2R-converter in real systems, one can see from the figures above that it can regenerates degraded signals even with low input powers $(<-12 \mathrm{dBm})$. Conclusively it is not necessary to use other amplifiers or SOAs to amplify the signal before the regenerator. It is important to comment that the SOA used as a booster and the EDFA were just used in this setup just to degrade the input signal.

\section{Wavelength detuning}

The wavelength detuning between the modulated input $\left(\lambda_{1}\right)$ and CW $\left(\lambda_{2}\right)$ signals is another factor which influences the XGM conversion. It is calculated as $\Delta \lambda=\lambda_{1}-\lambda_{2}$. The wavelength detuning also influences the regenerative effects, because the regeneration is associated to the conversion efficiency. The results shown so far were obtained for $\Delta \lambda=1 \mathrm{~nm}$, from 1550 $\mathrm{nm}$ to $1551 \mathrm{~nm}$. In this section, $\Delta \lambda$ is varied and the $\mathrm{Q}$-factor improvement is observed. For the results presented here, the output signal of the regenerator was attenuated to guarantee the same power level of the modulated input signal.

In order to analyze the detuning influence in the Q-factor improvement, the CW signal at $\lambda_{2}$ was kept fixed at $1551 \mathrm{~nm}$ due to the narrow-band filter used before the oscilloscope input. If the wavelength of this signal had been modified, it would be necessary adjustments of the filter for each case. Thus, the wavelength of the modulated input signal $\left(\lambda_{1}\right)$ was varied to obtain different detuning.

The results for the deterioration cases "SOA" and "LINK+SOA" are illustrated in the Figure 15 (a) for a 10.3 Gbps NRZ. For these cases, the power values of the CW and modulated input channels were kept fixed. Only the modulated signal wavelength $\left(\lambda_{1}\right)$ was varied. As seen in the Figure 3(c), a band-pass filter was used after the SOA used as a booster. As a consequence, the wavelength variation of the modulated input signal is limited by the filter bandwidth, which is about $4 \mathrm{~nm}$. Hence the detuning varies just from -3 to $1 \mathrm{~nm}$ in the curves of the Figure 15(a).

Analyzing the case "SOA", it is seen a $\Delta \mathrm{Q}$ decreasing while the detuning increases, with high $\Delta \mathrm{Q}$ values for the up-conversions. As stated before, although this case presents more extinction rate degradation, it causes more SOA gain saturation and can generate more regenerative effects. The $\Delta \mathrm{Q}$ values ranged from 1.6 to 2.4. Furthermore, there was a $\mathrm{Q}-$ factor improvement for conversions to the same wavelength, which is an interesting fact because the device presented here also can be used in a system where is necessary the signal regeneration without wavelength conversion.

The case "LINK+SOA" shows a behavior like the previous, but with higher $\Delta \mathrm{Q}$ values (2.5 to 4.2). The behavior of this case has a minimum for a 0 (zero) detuning (conversion to the same wavelength) with $\Delta \mathrm{Q}$ increasing again for positive detuning. It is not possible to show higher positive detuning values because of the band limiting of the filter, but due to the curves behavior, it is expected that $\Delta \mathrm{Q}$ also increases with the positive detuning increasing. By the behavior of these two cases, it is expected that for higher absolute values of detuning, the Q-factor improvements still have good results but there will be limitations for very large detuning values.

The Figure 15(b) presents the variation of the Q-factor improvement as a function of the wavelength detuning for the deterioration cases "EDFA", "LINK+EDFA" and "SOA+LINK+EDFA" for a 7 Gbps NRZ . The case "EDFA" has a behavior similar to that in 
the Figure 15(a) for the same detuning range from -3 to $1 \mathrm{~nm}$, with a $\Delta \mathrm{Q}$ decreasing while the detuning increases, and with a minimum for the conversion to the same wavelength, besides better results for up-conversion. However, without the band-pass filter cited previously, it was possible to extend the detuning values from -15 to $+15 \mathrm{~nm}$, observing that for high absolute values of detuning there is a $\Delta \mathrm{Q}$ decrease. Considerable values for detuning of $-15 \mathrm{~nm}(2.8)$ and $+15 \mathrm{~nm}$ (3.3) were attained even though with the $\Delta Q$ reduction. This deterioration case shows the highest $\Delta \mathrm{Q}$ values, varying from 2.8 to 4.8 .

The case "LINK+EDFA" has a slight different behavior, with higher $\Delta \mathrm{Q}$ values for downconversions. There is an almost continuous $\Delta \mathrm{Q}$ increasing with the wavelength detuning increasing, and the decrease occurs only for very high detuning values. In the curve of the case "LINK+EDFA" is also seen lower $\triangle \mathrm{Q}$ values, varying from 0.3 to 2.9. For the extreme cases, a Q-factor improvement was attained from 0.9 to $-5 \mathrm{~nm}$, and from 1.1 to $+10 \mathrm{~nm}$.

The case "SOA+LINK+EDFA"involves all the deterioration effects discussed and it is also showed in the Figure 15(b). The detuning values were limited by the band-pass filter. The behavior of this case is similar to the case "LINK+EDFA", with $\Delta \mathrm{Q}$ values ranging from 2.1 to 3.5 .

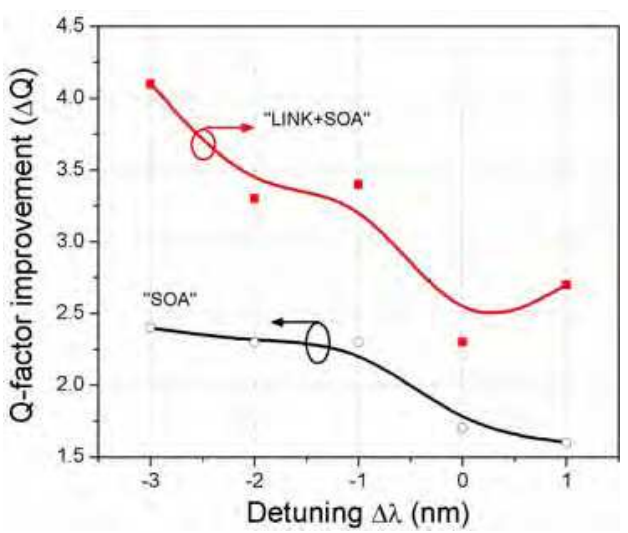

(a)

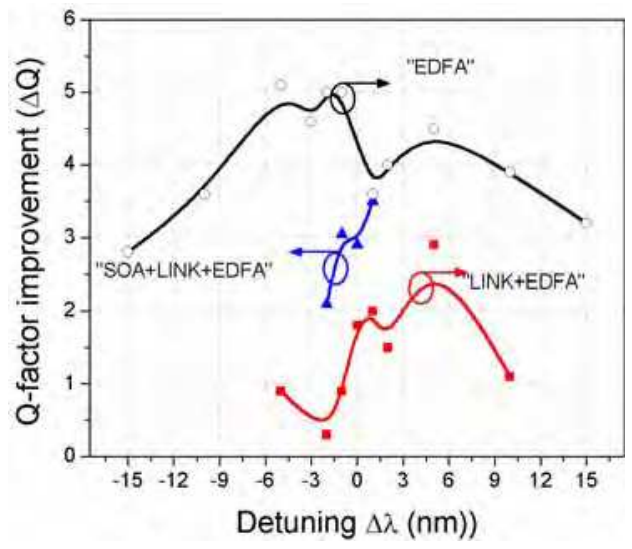

(b)

Fig. 15. Q-factor improvement versus detuning for the deterioration cases: (a) "SOA" and "LINK+SOA"; (b) "EDFA", "LINK+EDFA" and "SOA+LINK+EDFA".

In a broader context, it is possible to observe that $2 \mathrm{R}$-converter can keep the regenerative effects even for large detuning, assuming different behaviors in accordance with the deterioration case and showing better performance for up-conversion in some cases and for down-conversion in others. Good $\Delta \mathrm{Q}$ values were attained for conversions to the same wavelength, ranging from 1.6 to 4.2. Additionally, in the cases where a band-pass filter does not limit the detuning, there were good regenerative effects for up-conversion or downconversion for detuning up to $15 \mathrm{~nm}$.

\section{Bit rate}

High bit rates are interesting for any practical optical communication system. Therefore, it is necessary to study the response of the $2 \mathrm{R}$-converter presented in this chapter at distinct bit rates. For this study, the modulated input signal and the CW signal wavelengths were kept 
fixed at 1550 and $1551 \mathrm{~nm}$, respectively. Furthermore, the powers of these signals were also kept fixed and the regenerator output signal was not attenuated to the same power level of the modulated input signal. Hence, the signal re-amplification and re-shaping are considered in the results of this section.

Studies were conducted for the cases "SOA" and "LINK+SOA", with values ranging from 0.622 to 13.5 Gbps (Ribeiro et al., 2009a). The Figure 16(a) shows the Q-factor improvement as a function of the bit rate for the case "LINK+SOA". It is possible to observe that $\Delta \mathrm{Q}$ increases almost linearly with a bit rate around 5 Gbps. The best $\Delta \mathrm{Q}$ values ( $>4$ units) were obtained from 5 to $10.7 \mathrm{Gbps}$, showing a slight decreasing while the rate increasing. For values upper than 10.7 Gbps occur an abrupt $\Delta \mathrm{Q}$ decreasing until the minimum value 1 at 13.5 Gbps.

The analysis of the deterioration case "SOA" is illustrated in the Figure 16(b) with a similar behavior. The main differences are the extension of linear $\Delta \mathrm{Q}$ increasing up to $7 \mathrm{Gbps}$ and the lower $\Delta \mathrm{Q}$ values, with a maximum of 2.5 units. Lower $\Delta \mathrm{Q}$ values occur due to the low overshoots from the modulated input signal used in this analysis, which reduce the $\Delta Q$ improvement provided by the regenerator.

The behavior of those curves can be explained by the operation of the $2 \mathrm{R}$-converter. As seen before, the regenerator acts like a "low-pass filter". Hence, "high-frequency components", like the overshoot that arises in some cases, are filtered after the regenerator. The speed of the variance presented in each bit level varied with the modulation bit rate. Thus, for low frequencies (up to $5 \mathrm{Gbps}$ ) the speed of the variance is lower, and by the low-pass behavior of the regenerator variance is not very reduced, showing lower $\Delta \mathrm{Q}$ values. With the bit rate increasing, the speed of the variance is higher, what increases the variance reducing, thereby increasing $\Delta \mathrm{Q}$.

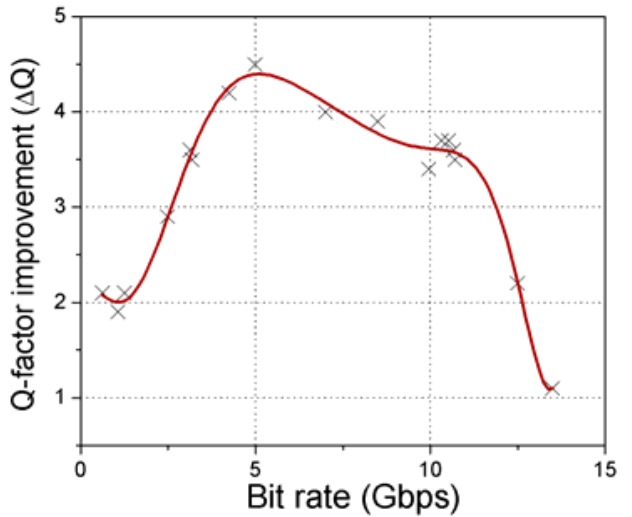

(a)

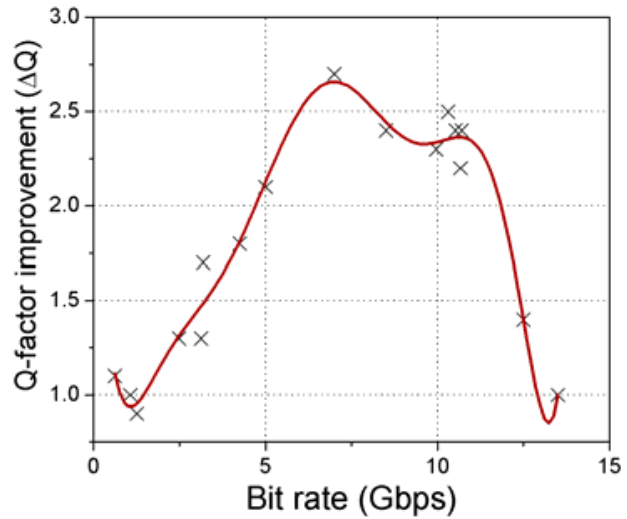

(b)

Fig. 16. Q-factor improvement versus bit rates for the deterioration cases: (a) "LINK+SOA" (adapted from Ribeiro et al., 2009a) and (b) "SOA".

In the range from 5 to $10.7 \mathrm{Gbps}, \Delta \mathrm{Q}$ has a little variation because speed of the variance at these rates is similar. But there is a small $\Delta \mathrm{Q}$ decreases in this range, influenced by another effect, which becomes relevant for rates upper than $10.7 \mathrm{Gbps}$, the pattern-dependence. At higher rates, the recovery time of the SOA gain is not enough to keep the bit pattern and distortion could occur in cases where there are large sequences of bits "1", for example. This 
pattern-dependence is stronger in the NRZ modulation case at higher rates. Thus, at rates upper than $10.7 \mathrm{Gbps}$, the pattern-dependence has more influence in the Q-factor improvement than the low-pass filter behavior, resulting in a reduction of the Q-factor improvement. The SOA gain recovery time is associated with the carrier lifetime in the SOA active region, which allows assign those bit rate limitations to the utilized SOA. To conclude if the limitation is either due to the SOA or it is from the setup of the 2R-converter used, it would be necessary another SOA with faster response. The discussion above is related only to the regenerator performance, which decays at rates upper than 10.7 Gbps. Despite this decrease in the $\mathrm{Q}$-factor improvement, there are positive $\Delta \mathrm{Q}$ for higher rates, confirming that the $2 \mathrm{R}$-converter regenerates even at rates above $10.7 \mathrm{Gbps}$.

\section{Conversion efficiency}

The device presented in this chapter reshapes the optical pulses simultaneously with the wavelength conversion. So, it is necessary an analysis about the conversion efficiency, which was done using the regenerator in a different setup from that one showed in the Figure 1. The results presented until this section were obtained using $13.5 \mathrm{Gbps}$ as the maximum bit rate. Then, in order to analyze the wavelength conversion efficiency, the modulation rate was augmented with a sinusoidal signals generator up to $20 \mathrm{GHz}$ (Ribeiro et al., 2009a), with corresponds to the bandwidth of a 40 Gbps NRZ signal.

Since the purpose of this section is the analysis of the wavelength conversion efficiency based on the XGM effect in a counter-propagating mode, the methods to degrade the input signal were not used in these measures. In order to study the conversion efficiency it is necessary to analyze the modulation index. To analyze the modulation frequency components, both the input and the output regenerator signals were converted to the electric field by a photodetector and observed in an electrical spectrum analyzer. As a result of the sinusoidal modulation, the spectrum shows only one peak in the modulation frequency. Thus, the conversion efficiency is determined as the difference between the values of this peak before and after the regenerator.

The results were obtained for two situations. In the first one, the modulated input signal and the CW signal were kept fixed at 1550 and $1551 \mathrm{~nm}$, respectively. Moreover, the CW carrier power was maintained at $-16 \mathrm{dBm}$ and the modulated signal input power was varied to values of $-16,-12.3$, and $-8.5 \mathrm{dBm}$. The efficiency conversion results for this case are illustrated in the Figure 17(a), where is seen conversion efficiency values up to $25 \mathrm{~dB}$ for a $-16 \mathrm{dBm}$ modulated signal input power. In addition, there is a conversion efficiency increase from 4 to $7 \mathrm{~dB}$ for values of the modulation frequency up to $4.5 \mathrm{GHz}$, and decreasing beyond this value. Still, there is positive conversion efficiency for power values lower than $-12.3 \mathrm{dBm}$, even at $20 \mathrm{GHz}$. The best conversion efficiency results were obtained for the lowest modulated signal powers, and they are associated with the SOA gain saturation, which is more intense for higher values of modulated signal power.

In the second situation, the modulated signal input power and the $\mathrm{CW}$ signal power were kept fixed in $-9.5 \mathrm{dBm}$ and $-9 \mathrm{dBm}$, respectively. However, the modulated signal wavelength was varied while the $\mathrm{CW}$ signal was fixed at $1551 \mathrm{~nm}$. The results of the conversion efficiency as a function of the modulation frequency are presented for distinct detuning values (already defined above as $\Delta \lambda=\lambda_{1}-\lambda_{2}$ ) in Figure 17(b). As the previous case, the efficiency increases up to $4.5 \mathrm{GHz}$, decreasing after this value. Nevertheless, positive conversion efficiency values occur for the most of detuning cases, except for the $-10 \mathrm{~nm}$ 
detuning. According to the results, there is higher conversion efficiency for downconversion, exhibiting very similar behavior between the +10 and $+5 \mathrm{~nm}$ detuning cases, with an efficiency peak of $15 \mathrm{~dB}$. The conversion efficiency for the detuning values of $-1,0$ and +1 have similar behavior at conversion efficiency ranging from $13 \mathrm{~dB}(4 \mathrm{GHz})$ to $5.5 \mathrm{~dB}$ $(20 \mathrm{GHz})$ (not shown here).

The good results attained for the conversion efficiency of the $2 \mathrm{R}$-converter show that this device can be used both as a regenerator and a wavelength converter. Furthermore, in some cases, good conversion efficiency up to $20 \mathrm{GHz}$ shows that this device will perform well as a wavelength converter even at high frequencies of modulation.

The behavior observed in Figure 17(a) and (b) may help to elucidate the results of the previous section. Clearly, the results of these figures indicate that the device has good results in wavelength conversion, and they are not related to regenerative features. Besides, the Figure 17(a) and (b) cases are quite different from the Figure 16(a) and (b). In the first case, it is a sinusoidal signal and input signal without deterioration. In the second, the signal is a PRBS pulse stream and the input signal is deteriorated. Despite the differences, there are common points in the results: an increase in the Q-factor improvement up to 5 Gbps and an increase in the conversion efficiency up to $4.5 \mathrm{GHz}$, followed by a decrease of these parameters. Thus, the behavior of $\Delta \mathrm{Q}$ with the bit rate can be said as dependent of the conversion efficiency, which complements the previous explanations about the regenerator behavior like a "low-pass filter", and the pattern-dependence effects. Despite this similarity, there is still some doubt whether the regenerator performance is limited by the SOA rate or the setup used, because from this section results it is just possible to know that as a converter, the device can gets good values of conversion efficiency up to $20 \mathrm{GHz}$ sinusoidal modulation, but nothing might be concluded from the regenerative features.

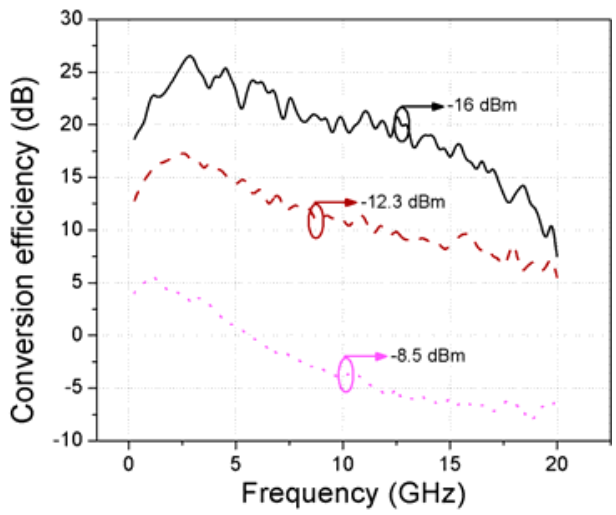

(a)

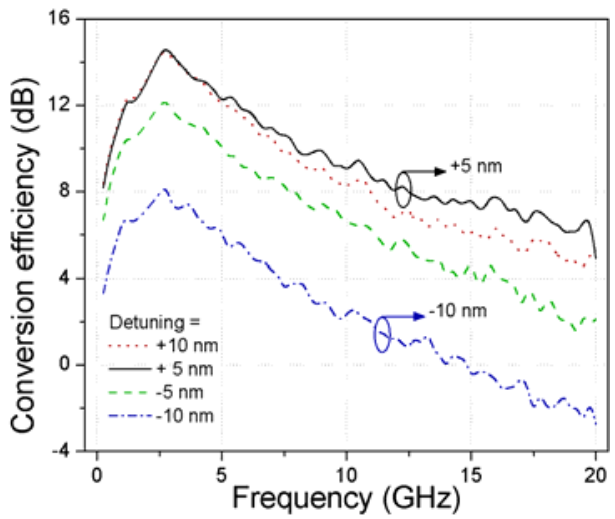

(b)

Fig. 17. Conversion efficiency versus frequency: (a) for fixed detuning and CW carrier power, varying the modulated signal optical power; (b) for fixed total input power, varying detuning (adapted from Ribeiro et al., 2009a).

\section{Bit error rate}

As observed in eye diagrams presented before, the bit reshape is visible and quantified by the Q-factor improvement. These results were analyzed only on the oscilloscope, without 
considering the real improvement that these results could cause in communications systems. In this way, it is interesting to study if the $2 \mathrm{R}$-converter can reduce the error of the receptor when it decides what kind of bit is arriving. The probability of incorrect identification of a bit by the decision circuit of the receiver is the BER definition.

The BER results showed in this work are just an estimation, because they were obtained from measured eye diagrams using equations, in which some approximations were assumed. The equations consider just the thermal noise, well described by Gaussian statistics with zero mean and the shot noise, with a ordinary approximation, treating it as a Gaussian random variable for both $\mathrm{p}-\mathrm{i}-\mathrm{n}$ and $\mathrm{APD}$ receivers, but with different variances. The sum of two Gaussian random variables (the two noise types considered) is also a Gaussian random variable. As a consequence the BER is given by (Agrawal, 2002):

$$
B E R=\frac{1}{4}\left[\operatorname{erfc}\left(\frac{I_{1}-I_{D}}{\sigma_{1} \sqrt{2}}\right)+\operatorname{erfc}\left(\frac{I_{D}-I_{0}}{\sigma_{0} \sqrt{2}}\right)\right]
$$

where $I_{1}$ and $I_{0}$ are the current level of the level ' 1 ' and ' 0 ', respectively; $\sigma_{1}$ and $\sigma_{0}$ are standard deviation of the level ' 1 ' and ' 0 ', respectively; $I_{D}$ is the decision threshold; and erfc stands for the complementary error function. Other approximation is $\ln \left(\sigma_{1} / \sigma_{0}\right)=0$.

The BER with optimum setting of the decision threshold is obtained by using (1) and (2) and depends only on the Q-factor as (Agrawal, 2002):

$$
B E R=\frac{1}{2} \operatorname{erfc}\left(\frac{Q}{\sqrt{2}}\right) \approx \frac{\exp \left(-Q^{2} / 2\right)}{Q \sqrt{2 \pi}}
$$

Given that the Q-factor can be obtained directly from eye diagrams visualized at the oscilloscope, it is possible to use (3) and obtain the BER estimation. As the BER values presented are just estimatives, lower values were obtained. Therefore, the graphics we presented illustrates only results that could be obtained in experiments (up to 10-13). Besides, the BER floor similar to one obtained in experiments is difficult to observe due to the lower BER values attained.

The analysis of the receptor bit error rate for different received signal power is necessary to study the BER estimation. In this way, the input BER estimation (before the 2R-converter) was calculated from the Q-factors attanied for different modulated input signal powers $\left(\lambda_{1}=1550 \mathrm{~nm}\right)$ for each deterioration cases. After the input BER estimation, the CW signal power $\left(\lambda_{1}=1551 \mathrm{~nm}\right)$ was optimized to obtain better values of output signal Q-factor, and consequently, better BER estimations. The output signal was attenuated to guarantee the same power level of the modulated input signal power, in order to compare the input and output BER estimations. The measurements were done at 7 Gbps NRZ.

The obtained results are illustrated in Figure 18, presented as - $\log (\mathrm{BER})$ versus the modulated signal input power that was the same level after the regenerator due to the use of an attenuator, and so was represented in the figures just as power. By the results of Figure 18 , the absence of low values and large variation of the modulated signal power can be observed. This fact is due to the insufficient receiver sensitivity of the used oscilloscope. In addition, the curves presented in Figure 18 are fitting by polynomials to better represent the bevahior of the attained points. 


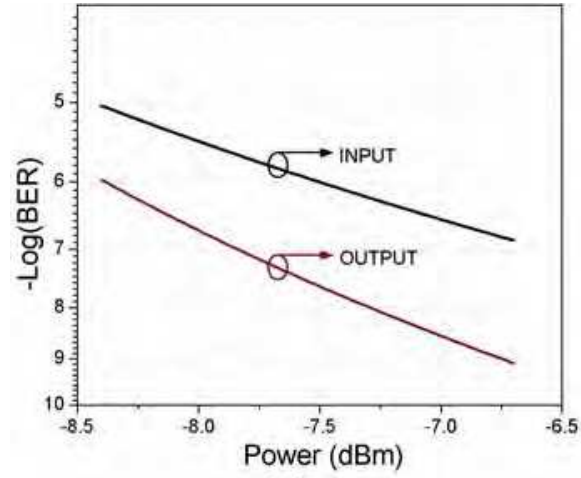

(a)

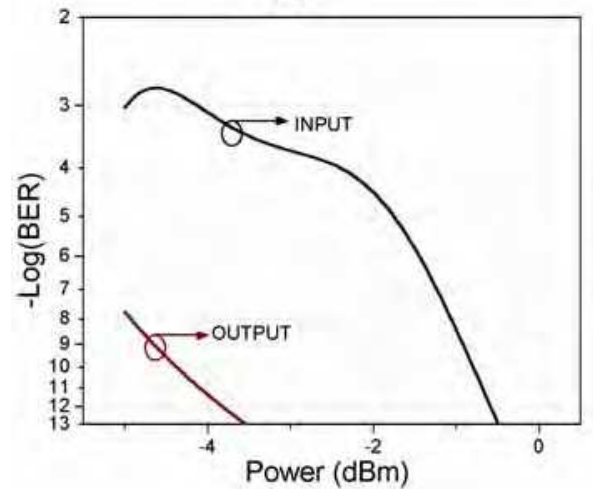

(c)

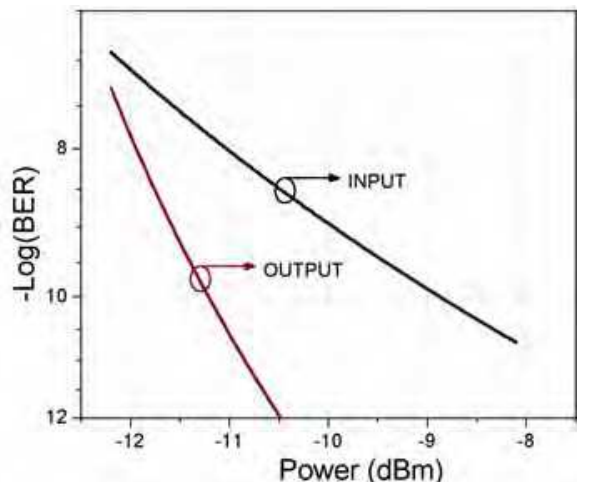

(b)

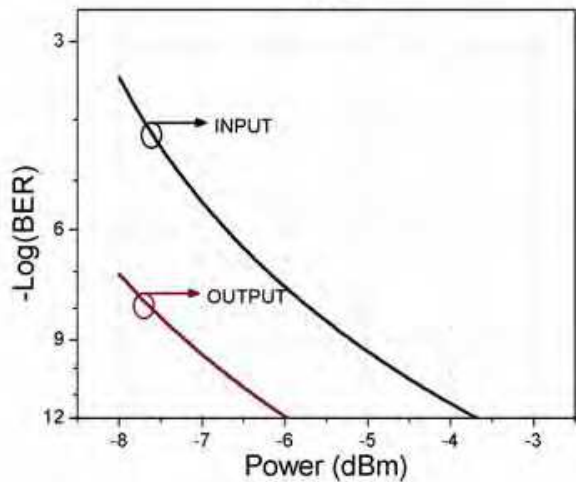

(d)

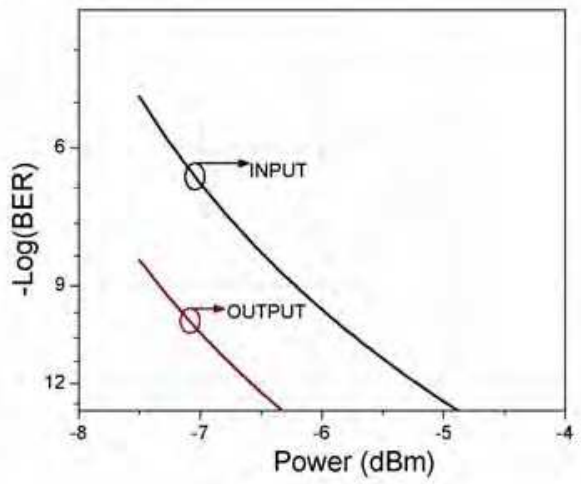

(e)

Fig. 18. BER estimation for different deterioration cases: (a) "SOA"; (b) "LINK+SOA"; (c) "EDFA"; (d) "LINK+EDFA", and (e) "SOA+LINK+EDFA".

The case "SOA" is illustrated in Figure 18(a). In this figure, it is observed an improvement of $1 \mathrm{~dB}$ in the power needed at the receiver in order to present the same BER, and BER values of 10-10. The case "LINK+SOA" is shown in Figure 18(b). For this case, low BER values were 
obtained due to the high Q-factor values and an improvement of $2 \mathrm{~dB}$ was obtained in the power level. Therefore, it could be note a trend that for higher power values, this improvement can be higher.

For the Figure 18(c) (case "EDFA"), it can be observed higher modulated signal power and a stranger behavior for the input BER estimation. The BER output corresponds to the expected BER estimation behavior, presenting BER values that starts in $10^{-8}$ due to the high Q-factor values obtained. An improvement of $4 \mathrm{~dB}$ in the power needed to have the same BER was obtained.

The performance of the case "LINK+EDFA" in Figure 18(d) is similar to the other cases with improvement of $2.5 \mathrm{~dB}$. The last case in Figure 18(e) involves the various deterioration types ("SOA+LINK+EDFA"). It can be observed an improvement of $1.5 \mathrm{~dB}$ in the power.

The BER estimation values presented here could be modified, depending on the modulated input signal deterioration. In this manner, the objective of this BER estimation is to compare the input and output BER results for each deterioration case, observing the improvement that the 2R-converter could provoked in optical systems, without comparison between different deterioration cases. As mentioned before, the BER values presented here are just an estimation calculated from Q-factor attained from measured eye diagrams, which justified the lower BER values. Excepting these lower BER values, the curves behavior (disregarding the BER input of the case "EDFA") and the improvement in power level to have the same BER value are feasible. Nevertheless, the study of extinction ratio (ER) is necessary since the BER analysis were obtained just from Q-factors. By considering a real system and measuring the BER experimentally, the ER migth be responsible for a BER results different from the presented ones in this section.

\section{Extinction ratio}

In the previous sections, the improvement of the bit format, of the Q-factor, and consequently of the BER estimation caused by the $2 \mathrm{R}$-converter was demonstrated. However, this regenerator is based on the XGM effect and so it presents ER deterioration. This figure of merit is defined as the relation between the power levels of the bits " 1 " and " 0 ". In this way, lower ER means that power levels of the bit " 1 " and " 0 " are near, being more difficult for the receptor make a decision about which bit was received. Besides, the ER is related to optical signal to noise ratio.

In Figure 19, the study of ER deteriorations after the regenerator for the all cases of deterioration present in this chapter are reported. This study was optimized just for the ER, others parameters were fixed, and the input ER was varied through the deviation of the modulator voltage. The ER input and output were obtained directly from the oscilloscope for the deterioration cases studied in this chapter. An up-conversion from 1550nm to $1551 \mathrm{~nm}$ was done to study the ER deterioration. As mentioned before, despite this type of wavelength conversion presents higher ER degeneration, it also presented higher SOA gain saturation, enabling higher regenerative effects.

The curves in Figure 19 were obtained by polynomial adjustment. It could be noted that there is an increase of the ER degeneration (input ER - output ER) with an input ER increase in all the deterioration cases. The case "LINK+EDFA" presents the better perfomance with lower ER degeneration values, and in some cases with ER improvement. Nevertheless, these ER improvements should be associated with small inaccuracies of the values obtained from the oscilloscope. 
As mentioned before, these ER degeneration results are higher because up-conversion was used. If down-conversion were used, lower ER degeneration values would be attained. The worst ER degeneration value obtanied was $5.35 \mathrm{~dB}$ for the deterioration case "LINK+SOA" and an input ER of $10.7 \mathrm{~dB}$.

Despite this deterioration, it is necessary to compare the ER degradation with the Q-factor improvement to validate the obtained results for BER estimation. Thus, if the Q-factor improvements $(\Delta \mathrm{Q})$, converted to $\mathrm{dB}$, present higher values than the ER degeneration, the obtained values of output BER will be higher than input BER values, proving the regenerative capacity of the $2 \mathrm{R}$-converter.

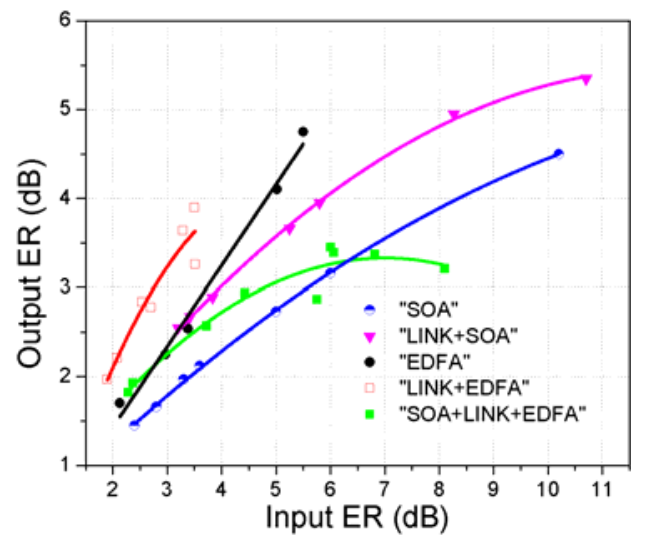

Fig. 19. Output ER as function of the input ER for different cases of input signal deterioration.

The Figure 20 shows the comparison between the Q-factor improvement $(\Delta \mathrm{Q})$ and the ER degeneration for different deterioration cases, considering different values of the output signal power, which were attenuated to guarantee the same values of the modulated input signal power. For the case "SOA" showed in Figure 20(a), it could be noted that the Q-factor improvement $(\Delta \mathrm{Q})$ is lower than ER degeneration for all the power values, differently of the case "LINK+SOA" (Figure 20(b)) in which, for the power values higher than $-10.8 \mathrm{dBm}$, the $\mathrm{Q}$-factor improvement $(\Delta \mathrm{Q})$ is higher than ER degeneration.

In Figure 20(c), the case "EDFA" is illustrated. In this figure, the Q-factor improvement surpass the ER degeneration for all power values as well as the case "LINK+EDFA"(Figure 20(d)). The difference between these cases is the higher Q-factor improvement for the first case. Moreover, some ER degeneration positive values were observed for the case "EDFA". Nevertheless, these results are affected by inaccuracies in the values obtained from the oscilloscope.

In Figure 20(e), the case "SOA+LINK+EDFA" is reported. This case presented a similar behavior with Figure 20(b), presenting power values where Q-factor improvement surpass ER deterioration and other values where this result does not occur. For the range of -7 to -4 $\mathrm{dBm}$, the $\Delta \mathrm{Q}$ values are higher then ER degeneration as well as the range of -2.7 to $-1.2 \mathrm{dBm}$. For power value between these ranges, the ER degeneration surpass $\Delta \mathrm{Q}$.

These obtanied results are relative to the used parameters. Note that for different modulated input signal power values, or even for different deterioration levels (input Q-factor) for the 
deterioration cases presented, the curves behavior could be different. One example is the case "SOA", for other deterioration level, the improvement Q-factor can surpass the ER degeneration.

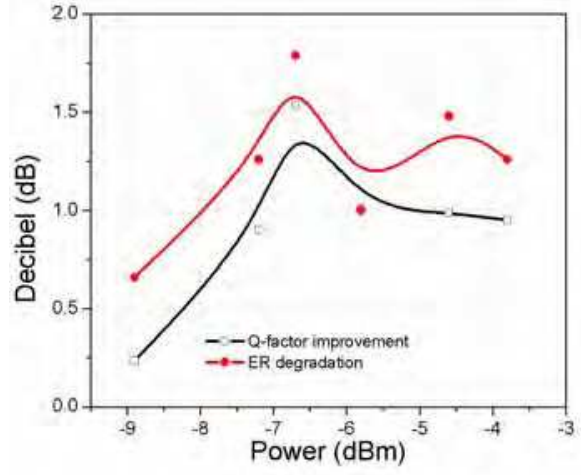

(a)

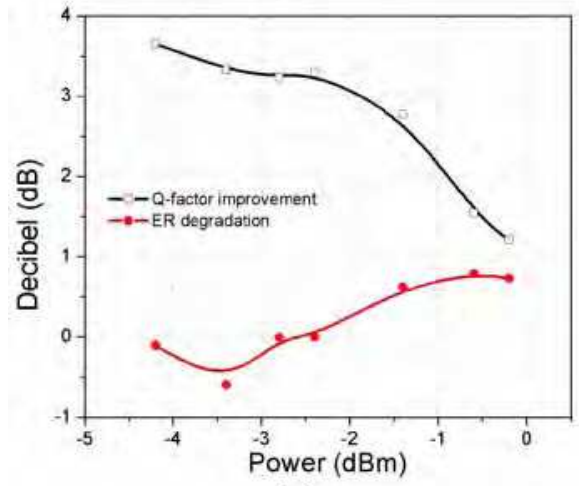

(c)

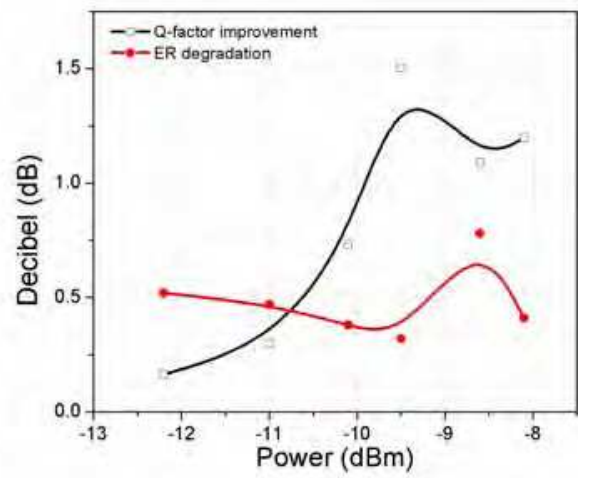

(b)

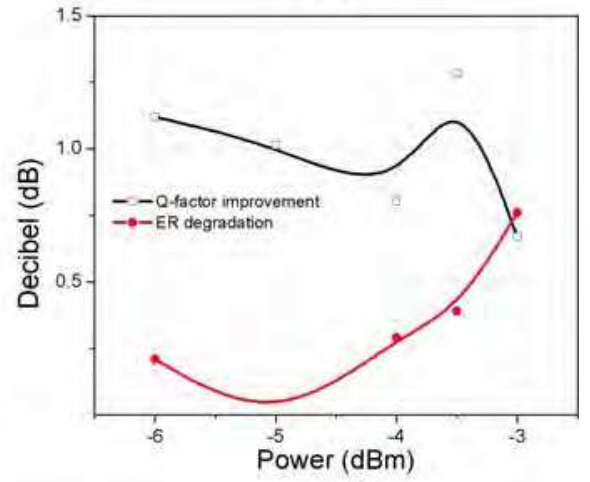

(d)

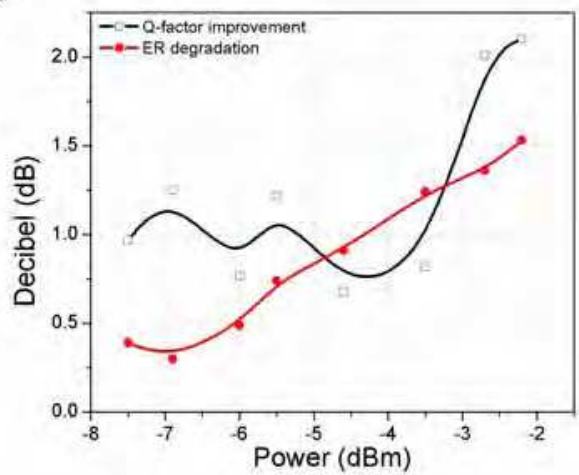

(e)

Fig. 20. Q-factor improvement versus ER degradation for different deterioration cases:

(a) "SOA"; (b) "LINK+SOA"; (c) “EDFA"; (d) "LINK+EDFA", and (e) "SOA+LINK+EDFA". 
In this manner, observing these comparison between Q-factor improvement and ER degeneration, it is possible to prove that, in the most of the cases, the output BER (despite the decrease of the BER values due to ER degeneration not considered) will be higher than input BER, confirming the regenerative effects presented by the $2 \mathrm{R}$-converter.

\section{Integration possibility}

A great advantage to use setups based on SOA is the integration capacity of this amplifier due to its small dimensions. There are many devices that use SOA integrated to other optical components as for example: wavelength conversion based on SOA-MZI (Kehayas et al., 2006), regenerator and wavelength converter using SOA integrated to distributed-feedback laser (Stephens et al., 1999), optical clock recovery with SOA integrated to mode-locking laser (Koch et al., 2007), and others.

The 2R-converter is very simple, using just one non-linear SOA, an optical circulator, an optical isolator and a CW laser. The integration between SOA and laser is already used as the examples mentioned before. Integrated optical circulators that use nonreciprocal phase shifters already exist a long time ago (Okamura et al., 1984). In addition, there is waveguide polarization-independent optical circulator using MZI (Sugimoto et al., 1999) or MMI with Faraday rotator (Zaman et al., 2006). Optical isolator also been obtained using nonreciprocal coupled waveguides (Bahlmann et al., 1999), based on efficient nonreciprocal radiation mode conversion (Shintaku, 1998) or other ways. Besides, if the narrow filter used to reduce the ASE noise before the oscilloscope input is considered essential to the $2 \mathrm{R}$-converter, there are optical filters based on ring resonators (Rabus et al., 2002), on MZI with two ring resonators (Rasras et al., 2007), and on photonic crystals (Zhang et al., 2007). In this section, just some techniques used to obtain the integration of the optical components mentioned are present, existing others technique not reported in this chapter.

The results mentioned about SOA, laser, optical circulator, isolator, and filter do not considered the simultaneously integration of these several components. In this manner, it is necessary the study of techniques to obtain the integration of these different optical component like the experimental setup in Figure 1 to prove the $2 \mathrm{R}$-converter integration possibility. Thus, optical components integration area should be asserted higher integration possibility to the $2 \mathrm{R}$-converter.

\section{Conclusion}

The 2R-converter presented in this chapter demonstrated good performance for regeneration and wavelength conversion of signals deteriorated by different effects. Considering these different deterioration cases, the $2 \mathrm{R}$-converter presented improvement in the degenerated signal as: signal amplification with an optical gain of $12 \mathrm{~dB}$, reduction of the ASE noise presented in bit levels " 1 " and " 0 " with variance decreasing, overshoot elimination and reduction of the intrinsic dispersion degenerative effects. These results were quantified by the $\mathrm{Q}$-factor improvement $(\Delta \mathrm{Q})$ presenting values up to 4.5 .

Furthemore, an existence of an optimum relation between the power of modulated input signal and the $\mathrm{CW}$ signal that maximize the regenerative effects was observed. This relation is associated to the SOA gain saturation level. Regeneration for lower input power $(<-12$ $\mathrm{dBm}$ ) was observed too. Additionally, the $2 \mathrm{R}$-converter demonstrated to be practically independent of the polarization angle of modulated input signal with gain variation of just 
$0.9 \mathrm{~dB}$. Regeneration was obtained for up-conversion, down-conversion and conversion to the same wavelength, with good results even for large detuning $(15 \mathrm{~nm})$.

The $2 \mathrm{R}$-converter presented good conversion efficiency values for rate of $20 \mathrm{GHz}$ sinusoidal. In relation to the regenerative effects, the device presented good results up to $13.5 \mathrm{Gbps}$ NRZ with better perfomance from 5 to10.7 Gbps. The use of another SOA with lower gain recovery time is a possiblity to future works to determine if the bit rate limitation is caused by the used SOA or by the experimental setup configuration.

The BER estimations from measured eye diagrams were presented, confirming the good 2R-converter performance. Good results were obtained for different cases of input signal deterioration with improvement in the receiver sensitivity up to $4 \mathrm{~dB}$. Moreover, for most cases, the improvement caused by the $2 \mathrm{R}$-converter surpass the ER degeneration, which is presented in all wavelength conversion based on XGM effect.

In this way, the $2 \mathrm{R}$-converter showed good regenerative capacity for different deterioration types. Due to the use of a SOA, this setup can be integrated, decreasing the cost and volume. Another important features of the $2 \mathrm{R}$-converter are: simplicity; robust operation; high-speed reconfiguration, limited just by the $\mathrm{CW}$ laser tuning (since the narrow filter in the oscilloscope input is not considered). In this manner, wavelength conversion and $2 \mathrm{R}-$ regeneration in a simple scheme with SOA was presented and characterized.

\section{Acknowledgments}

This work was supported by the Brazilian agency FAPESP (Fundação de Amparo à Pesquisa do Estado de São Paulo), under CEPOF and KyaTera-FAPESP project, and by CNPq.

\section{References}

Agrawal, G.P. (2002). Fiber-optic communication systems, Willey-Interscience, ISBN 0-47121571-6, New York, USA.

Bahlmann, N.; Lohmeyer, M.; Zhuromskyy, O.; Dotsch, H. \& Hertel, P. (1999). Nonreciprocal coupled waveguides for integrated optical isolators and circulators for TM-modes," Elsevier Optics Communications, Vol. 161, (March 1999) pp. 330337, DOI: 10.1016/S0030-4018(99)00027-9.

Chayet, H.; Ezra, S.B.; Shachar, N.; Tzadok, S.; Tsadha, S. \& Leuthold, J. (2004). Regenerative all-optical wavelength converter based on semiconductor optical amplifier, Proceedings of the Conference on Lasers and Electro-Optics (CLEO 2004), ISBN: 1-55752772-5, Baltimore-USA, February 2004.

Conforti, E.; Bordonalli, A.C.; Ho, S. \& Kang, S. (1999). Optical 2R remodulator using feedforward control of semiconductor optical amplifier, Microwave and Optical Technology Letters, Vol. 21, No. 1, (March 1999) pp. 39-42.

Contestabile, G.; Proietti, R.; Calabretta, N. \& Ciaramella, E. (2005). Reshaping capability of cross-gain compression in semiconductor amplifiers, IEEE Photonics Technology Letters, Vol. 17, No. 12, (December 2005) pp. 2523-2525, ISSN: 1041-1135.

Durhuus, T.; Mikkelsen, B.; Joergensen, C.; Danielsen, S.L. \& Stubkjaer, K.E. (1996). Alloptical wavelength conversion by semiconductor optical amplifiers, Journal of Ligthwave Technology, Vol. 14, No. 6, (June 1996) pp. 942-954, ISSN: 0733-8724.

Fischer, S.; Dulk, M.; Gamper, E.; Vogt, W.; Gini, E.; Mechior, H.; Hunziker, W.; Nesset , D. \& Ellis, A.D. (1999). Optical 3R regenerator for $40 \mathrm{Gbit} / \mathrm{s}$ networks, Electronics Letters, (November 1999), Vol. 35, No. 23, ISSN: 0013-5194. 
Funabashi, M.; Zhu, Z.; Pan, Z., Paraschis, L. \& Yoo, S.J.B. (2006). Optical clock recovery and $3 \mathrm{R}$ regeneration for $10-\mathrm{Gb} / \mathrm{s}$ NRZ signal to achieve 10 000-hop cascadability and 1 000 000-km transmission, IEEE Photonics Technology Letters, Vol. 18, No. 20, (December 2006) pp. 2078-2080, ISSN: 1041-1135.

Gallep, C.M. \& Conforti, E. (2002). Reduction of semiconductor optical amplifier switching times by preimpulse step-injected current technique, IEEE Photonics Technology Letters, Vol. 14, No. 7, (July 2002) pp. 902-904, ISSN: 1041-1135.

Kehayas, E.; Tsiokos, D.; Bakopoulos, P.; Apostolopoulos, D.; Petrantonaski, D.; Stampoulidis, L.; Poustie, A.; McDougall, R.; Maxwell, G.; Liu, Y.; Zhang, S.; Dorren, H.J.S.; Seoane, J.; Nielsen, P.H.; Jeppesen, P. \& Avramopoulos, H. (2006). $40 \mathrm{~Gb} / \mathrm{s}$ all-optical processing systems using hybrid photonic integration technology, Journal of Ligthwave Technology, Vol. 24, No. 12, (December 2006) pp. 4903-4911, ISSN: 0733-8724.

Kelly, A.E. (2001). Ultra high-speed wavelength conversion and regeneration using semiconductor optical amplifiers, Proceedings of the Optical Fiber Communication Conference (OFC 2001), ISBN:1-55752-655-9, Anaheim-USA, March 2001.

Koch, B.R.; Barton, J.S.; Masanovic, M.; Hu, Z.; Bowers, J.E. \& Blumenthal, D.J. (2007). Monolithic mode-locker laser and optical amplifier for regenerative pulsed optical clock recovery, IEEE Photonics Technology Letters, Vol. 19, No. 9, (May 2007) pp. 641643, ISSN: 1041-1135.

Leuthold, C.M.; Marom, D.; Cabot, S.; Ryf, R.; Bernasconi, P.; Baumann, F.; Jaques, J.; Neilson, D.T. \& Giles, C.R. (2003). All-optical wavelength converter based on a pulse reformatting optical filter, Proceedings of the Optical Fiber Communication Conference (OFC 2003), ISBN: 1-55752-746-6, March 2003.

Liu, Y.; Herrera, J.; Raz, O.; Tangdiongga, E.; Ramos, F.; Marti, J.; Waadt, H.; Koonen, A.M.J.; Khoe, G.D. \& Dorren, H.J.S. (2007). 160 Gbits/s all optical SOA based wavelength conversion and error-free transmisssion through two $50 \mathrm{~km}$ fibre links, Electronics Letters, Vol. 43, No. 25, (December 2007).

Merlier, J.; Morthier, G.; Caenegen, T.; Baets, R.; Moerman, I. \& Daele, P. (2001). Experimental demonstration of $15 \mathrm{~dB}$ extinction ratio improvement in a new $2 \mathrm{R}$ optical regenerator based on an MMI-SOA, Proceedings of the European Conference on Optical Communication (ECOC 2001), ISBN: 0-7803-6705-7, Amsterdam, Vol. 4, pp. 574-575. February 2008.

Okamura, Y.; Negami, T. \& Yamamoto, S. (1984). Integrated optical isolator and circulator using nonreciprocal phase shifters: a proposal, Appied Optics, Vol. 23, No. 11, (June 1984) pp 1186-1189, DOI:10.1364/AO.23.001886.

Ohman, F.; Bischoff, S.; Tromborg, B. \& Mork, J. (2003). Semiconductor devices for all-optical regeneration, Proceedings of the International Conference on Transparent Optical Networks (ICTON' 03), Vol. 2, pp-41-46, ISBN: 0-7803-7816-4, June 2003.

Rabus, D.G.; Hamacher, M.; Troppenz, U. \& Heidrich, H. (2002). Optical filters based on ring resonators with integrated semiconductor optical amplifiers in GaInAsP-InP, IEEE Journal of Selected Topics in Quantum Electronics, Vol. 8, No. 6, (November/December 2002) pp.1405, ISSN: 1077-260X.

Rasras, M.S.; Gill, D.M.; Patel, S.S.; Tu, k.y.; Chen, Y.K.; White, A.E.; Pomerene, A.T.S; Carothers, D.N.; Grove, M.J.; Sparacin, D.K.; Michel, J.; Beals, M.A. \& Kimerling, L.C. (2007). Demonstration of a fourth-order pole-zero optical filter integrated 
using CMOS processes, Journal of Ligthwave Technology, Vol. 25, No. 1, (January 2007) pp. 87, ISSN: 0733-8724.

Raz, O.; Herrera, J.; Calabretta, N.; Tangdiongga, E.; Anantathanasarn, S.; Notzel, R. \& Dorren, H.J.S. (2008). Non-inverted multiple wavelength converter at $40 \mathrm{Gbits} / \mathrm{s}$ using $1550 \mathrm{~nm}$ quantum dot SOA, Electronics Letters, Vol. 44, No. 16, (July 2007).

Ribeiro, N.S.; Gallep, C.M. \& Conforti, E. (2008). Wavelength conversion and 2Rregeneration using one semiconductor optical amplifier with cross-gain modulation compression, Proceedings of the Conference on Lasers and Electro-Optics (CLEO 2008), San jose -USA, ISBN:978-1-55752-859-9, May 2008.

Ribeiro, N.S.; Gallep, C.M. \& Conforti, E. (2009a). High eye-quality improvement by a single SOA regenerative wavelength converter, accepted to Microwave and Optical Technology Letters.

Ribeiro, N.S.; Toazza, A.L.; Gallep, C.M. \& Conforti, E. (2009b). Rise time and gain fluctuations of an electrooptical amplified switch based on multi pulse injection in semiconductor optical amplifiers, IEEE Photonics Technology Letters, Vol. 21, No. 10, (2009) pp. 769-771, ISSN: 1041-1135.

Shintaku, T. (1998). Integrated optical isolator based on efficient nonreciprocal radiation mode conversion, Applied Physics Letters, Vol. 73, No. 14, (October 1998), DOI: 10.1063/1.122331.

Simon, J.C.; Gay, M.; Bramerie, L.; Roncin, V.; Joindot, M.; Chartier, T.; Lobo, S.; Girault, G.; Le, Q.T.; Nguyen, T.N. \& Ngo, M.N. (2008). Long distance using optical regeneration, Proceedings of the Optical Fiber Communication Conference (OFC 2008), ISBN: 978-1-55752-856-8, San Diego-USA, February 2008.

Simos, H.; Bogris, A. \& Syvridis, D. (2004). Investigation of a 2R all-optical regenerator based on four-wave mixing in a semiconductor optical amplifier, Journal of Ligthwave Technology, Vol. 22, No. 2, (February 2004) pp. 595-604, ISSN: 0733-8724.

Stephens, M.F.C.; Penty, R.V. \& White, I.H. (1999). All-optical regeneration and wavelength conversion in an integrated semiconductor optical amplifier/distributed-feedback laser, IEEE Photonics Technology Letters, Vol. 11, No. 8, (August 1999) pp. 979-981, ISSN: 1041-1135.

Sugimoto, N.; Shintaku, T.; Tate, A.; Terui, H.; Shimokozono, M.; Kubota, E.; Ishii, M. \& Inoue, Y. (1999). Waveguide polarization-independent optical circulator, IEEE Photonics Technology Letters, Vol. 11, No. 3, (March 1999) pp. 335-337, ISSN: 10411135.

Zaman, T.R.; Guo, X. \& Ram, R.J. (2006). Proposal for a polarization-independent integrated optcial circulator, IEEE Photonics Technology Letters, Vol. 18, No. 12, (June 2006) pp. 1359-1361, ISSN: 1041-1135.

Zhang, Z.; Dainese, M.; Wosinski, L.; Xiao, S. \& Qiu, M. (2007). Optical filter based on twodimension photonic crystal surface-mode cavity in amorphous silicon-on-silica structure, Applied Physics Letters, Vol. 90, No. 4, (January 2007) pp. O41108-1, DOI: $10.1063 / 1.2432228$

Wang, J.P.; Savage, S.J.; Robinson, B.S.; S.A. Hamilton, S.A; Ippen, E.P; Mu, R.; Wang, H.; Spiekman, L. \& Stefanov, B.B. (2007). Regeneration using SOA-MZI in a 100 pass 10,000-km recirculating fiber loop, Proceedings of the Conference on Lasers and ElectroOptics (CLEO 2007), ISBN: 978-1-55752-834-6, Baltimore -USA, May 2007. 


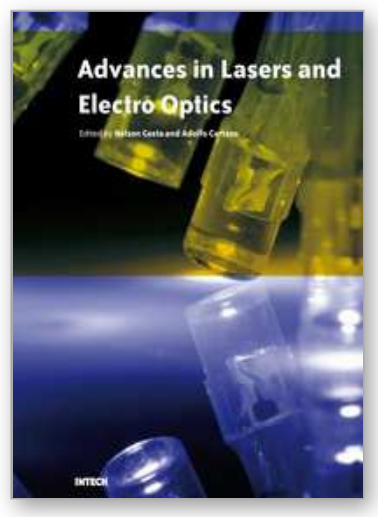

\author{
Advances in Lasers and Electro Optics \\ Edited by Nelson Costa and Adolfo Cartaxo
}

ISBN 978-953-307-088-9

Hard cover, 838 pages

Publisher InTech

Published online 01, April, 2010

Published in print edition April, 2010

Lasers and electro-optics is a field of research leading to constant breakthroughs. Indeed, tremendous advances have occurred in optical components and systems since the invention of laser in the late $50 \mathrm{~s}$, with applications in almost every imaginable field of science including control, astronomy, medicine, communications, measurements, etc. If we focus on lasers, for example, we find applications in quite different areas. We find lasers, for instance, in industry, emitting power level of several tens of kilowatts for welding and cutting; in medical applications, emitting power levels from few milliwatt to tens of Watt for various types of surgeries; and in optical fibre telecommunication systems, emitting power levels of the order of one milliwatt. This book is divided in four sections. The book presents several physical effects and properties of materials used in lasers and electro-optics in the first chapter and, in the three remaining chapters, applications of lasers and electro-optics in three different areas are presented.

\title{
How to reference
}

In order to correctly reference this scholarly work, feel free to copy and paste the following:

Napoleao S. Ribeiro, Cristiano M. Gallep, and Evandro Conforti (2010). Wavelength Conversion and 2RRegeneration in Simple Schemes with Semiconductor Optical Amplifiers, Advances in Lasers and Electro Optics, Nelson Costa and Adolfo Cartaxo (Ed.), ISBN: 978-953-307-088-9, InTech, Available from:

http://www.intechopen.com/books/advances-in-lasers-and-electro-optics/wavelength-conversion-and-2rregeneration-in-simple-schemes-with-semiconductor-optical-amplifiers

\section{INTECH}

open science | open minds

\section{InTech Europe}

University Campus STeP Ri

Slavka Krautzeka 83/A

51000 Rijeka, Croatia

Phone: +385 (51) 770447

Fax: +385 (51) 686166

www.intechopen.com

\section{InTech China}

Unit 405, Office Block, Hotel Equatorial Shanghai

No.65, Yan An Road (West), Shanghai, 200040, China

中国上海市延安西路65号上海国际贵都大饭店办公楼 405 单元

Phone: +86-21-62489820

Fax: $+86-21-62489821$ 
(C) 2010 The Author(s). Licensee IntechOpen. This chapter is distributed under the terms of the Creative Commons Attribution-NonCommercialShareAlike-3.0 License, which permits use, distribution and reproduction for non-commercial purposes, provided the original is properly cited and derivative works building on this content are distributed under the same license. 